\title{
Inequities in access to healthcare: analysis of national survey data across six Asia-Pacific countries
}

\author{
Samantha B Meyer ${ }^{*}$, Tini CN Luong ${ }^{\dagger}$, Loreen Mamerow ${ }^{\dagger}$ and Paul R Ward ${ }^{\dagger}$
}

\begin{abstract}
Background: Evidence suggests that there is a link between inequitable access to healthcare and inequitable distribution of illness. A recent World Health Organization report stated that there is a need for research and policy to address the critical role of health services in reducing inequities and preventing future inequities. The aim of this manuscript is to highlight disparities and differences in terms of the factors that distinguish between poor and good access to healthcare across six Asia-Pacific countries: Australia, Hong Kong, Japan, South Korea, Taiwan, and Thailand.
\end{abstract}

Methods: A population survey was undertaken in each country. This paper is a secondary analysis of these existing data. Data were collected in each country between 2009 and 2010. Four variables related to difficulties in access to healthcare (distance, appointment, waiting time, and cost) were analysed using binomial logistic regression to identify socio- and demographic predictors of inequity.

Results: Consistent across the findings, poor health and low income were identified as difficulties in access. Country specific indicators were also identified. For Thailand, the poorest level of access appears to be for respondents who work within the household whereas in Taiwan, part-time work is associated with difficulties in access. Within Hong Kong, results suggest that older (above 60) and retired individuals have the poorest access and within Australia, females and married individuals are the worst off.

Conclusion: Recognition of these inequities, from a policy perspective, is essential for health sector policy decision-making. Despite the differences in political and economic climate in the countries under analysis, our findings highlight patterns of inequity which require policy responses. Our data should be used as a means of deciding the most appropriate policy response for each country which includes, rather than excludes, socially marginalised population groups. These findings should be of interest to those involved in health policy, but also in policy more generally because as we have identified, access to health care is influenced by determinants outside of the health system.

Keywords: Equity, Access, Healthcare, Asia, Pacific, Social determinants, Policy

\footnotetext{
* Correspondence: samantha.meyer@flinders.edu.au

${ }^{\dagger}$ Equal contributors

Discipline of Public Health, Flinders University, Sturt Road, Bedford Park,

South Australia 5042, Australia
}

\section{Biomed Central}

(c) 2013 Meyer et al.; licensee BioMed Central Ltd. This is an Open Access article distributed under the terms of the Creative Commons Attribution License (http://creativecommons.org/licenses/by/2.0), which permits unrestricted use, distribution, and reproduction in any medium, provided the original work is properly cited. 


\section{Introduction}

Both trans-nationally [1-3] and within particular countries [4], it is widely recognised that public health policy and practice needs to focus on addressing the Social Determinants of Health (SDH) in order to increase the health of the most vulnerable and disadvantaged groups [5]. The focus on promoting health and preventing illness for the most vulnerable groups in society has been part of the named agenda of public health for decades, and is central to contemporary global agreements such as the Jakarta Declaration [6] and the Bangkok Charter for Health Promotion [7]. Indeed, Professor Sir Michael Marmot recently referred to health inequities as a "stain on our society" which requires concerted political will and moral imperative to change [8].

Equitable access to healthcare services is a major SDH $[1,3]$, with a WHO report stating there is a need for research and policy to address the critical role of "health services in reducing ill health and suffering, redressing inequities, and preventing future inequities" [9]. The WHO Commission on the SDH provides evidence on the link between inequitable access to healthcare and the resulting inequitable distribution of illness and make a recommendation for interventions to increase the equity of access to healthcare services [10-12].

There is a large research literature demonstrating that access to, quality of, and outcomes from healthcare are inequitable across a number of clinical areas including screening for a variety of cancers [13-16], surgical interventions [17-19] and primary care prescribing [20-22]. These examples provide evidence for the inverse care law' [23], whereby the groups with the greatest healthcare need receive the lowest levels of service. This evidence has led to an international call for action to reduce inequities in health and healthcare $[1,24,25]$.

The incorporation of equity in health provision helps to identify the significant roles of political and social consciousness in developing and sustaining the needs and agendas of all people in a fair and just way [26]. For the purposes of this manuscript, a key distinction needs to be made between a relatively large field of research which defines equity in health [27-30] and an emerging field of research which defines equity in healthcare [31-34]. It is hypothesised that improving equity in access to healthcare will lead to improved equity in health outcomes [11].

This manuscript presents for the first time, a secondary analysis of cross-country data which identifies populations at risk of poor access to healthcare in six Asia-Pacific countries: Australia, Hong Kong, Japan, South Korea, Taiwan, and Thailand. Specifically, this manuscript reports the findings of a population survey undertaken in each country regarding difficulties in access to healthcare: namely, difficulties due to distance, obtaining an appointment, waiting times and costs. The aim of this manuscript is to highlight disparities and differences in terms of the factors that seem to be important to distinguishing between poor and good access to healthcare within each country.

\section{Background}

Healthcare is socially determined in that it is influenced by social policies that govern the receipt and utilization of health services, allocation of healthcare resources, the financing of healthcare, and the quality of healthcare services [35]. Ensuring that appropriate resources are mobilized to meet the healthcare needs of different groups in different populations is key to healthcare access [36]. The notion of 'equity' is inextricably linked to the concept of access to healthcare, because the inclusion of fairness or social justice is one of the key factors necessary for the effective provision of healthcare [37].

There is a great deal of research focused on defining equity in access to healthcare [36,38-40], and its operationalisation [41-44]. The literature on "equity" and "access" to healthcare is abundant, diverse, and complex and the conceptualisation and operationalisation of these two concepts is not consistent [44]. Many studies have typically relied on measures of utilization as the basis for analyses of equity of access, although this is methodologically and conceptually problematic because it does not measure need and thus, does not identify problems of inequity. This study diverts the focus away from measuring utilization of health services and instead, measures socio-demographics against healthcare access indicators. We then discuss these findings in relation to healthcare provision within each of these six countries.

We conceptualise access as the level of facilitation or inhibition affecting the ability of individuals to gain entry to and receive care from healthcare services [45]. The accessibility of healthcare depends on a multitude of supply- and demand-side factors [46]. Supply-side factors include the distribution of facilities, waiting times, and human resources and capital $[36,47,48]$. Factors influencing accessibility on the demand-side result from predisposing, enabling, and needs factors [49], including socio-demographics, past experiences with healthcare, perceptions regarding health and illness, income levels and scope, and extent of health insurance coverage [50]. Our conceptualisation of access was used to guide our investigation which looks specifically at the supply-side factors affecting access (cost, waiting time, appointment, distance) and relevant demand-side factors (socio-demographic and health related predictors).

An equitable health service implies that individuals' utilisation of and access to service depends on their health state alone, and not upon their socio-economic status [51]. The research presented herein is based on 
our argument that this notion should be extended and access should not be based upon age, race, geography, gender, language, culture, and functional capacity. Equity in healthcare should be concerned with the reduction of systematic discrimination and marginalization between different groups and within groups, regardless of whether a group is found to attain a generally high or low level of equitable access. Our use of socio-demographic variables (e.g. sex, age, income) and variables related to the beliefs and expectations about health and illness (e.g. chronic health condition, self-rated health) help to reveal the differing levels and standards of needs, and have been included for analysis in this study.

\section{Methods}

The data presented in this manuscript come from a larger survey designed to investigate social quality [52] across six Asia-Pacific counties: Australia, Hong Kong, Japan, South Korea, Taiwan, and Thailand. The survey was developed, and data collected, by academic representatives from each of the universities involved; Australia (Flinders University), Hong Kong (Chinese University Hong Kong), Japan (Chiba University), Korea (Seoul National University), Taiwan (National Taiwan University), Thailand (King Prajadhipok Institute). The survey was then translated into the language of the host country and validated. The survey was then piloted in each language and tested for reliability (see [53] for an overview of the validity and reliability testing in Australia). All questions asked were the same, but in a different language. The authors undertook data collection in Australia only and therefore, this paper reports a secondary analysis of a combined dataset representing all countries involved. The merging and cleaning of the dataset was conducted by academics at Seoul National University. Details of method for selected countries represented in this paper are published elsewhere [54-57]. Data were collected in each country between 2009 and 2010.

Four variables related to difficulties in access to healthcare (distance, appointment, waiting time, and cost) were selected and form the basis of this manuscript. The survey question regarding access is provided in Table 1.

Ten independent variables (sex, age, marital status, work status, income, financial situation in the last year, subjective health satisfaction, self-rated health, perception of importance of health, and chronic health condition) were tested against the four dependent variables based on previous studies linking their relevance to equity of access to healthcare $[47,58,59]$.

Data were analysed using the SPSS version 17.0 (SPSS Inc., Chicago, IL, USA). Binomial logistic regression models were used to investigate associations for all six countries [60]. In order to conduct binomial regression, the original four dependent variables were recoded into new variables with two categories of response. 'Very difficult' and 'a little difficult' were recoded as 'difficult'. Responses 'not applicable/never needed to see a doctor' were coded as missing. 'Not difficult at all' was not recoded. Due to differences in data collection methods, and changes to survey questions to make them culturally relevant, a few of the independent variables were not available from some countries thus reducing the number of association tests performed. Goodness-of-fit for all models were checked $[60,61]$.

To ensure that there would be no redundant calculations during the multivariate analyses, co linearity diagnostics (using SPSS) were performed to check for variables that may have similar degrees of variance [62]. A tolerance value of $\leq 0.20$ and a variance inflation factor of $\geq 10$ were used to indicate a multicollinearity problem [63]. Diagnostics were conducted for all 24 models (six countries by four dependent variables).

Some methodological issues were faced when the data from all six countries were checked for consistency. Rice et al. (2010:82) identify that the design of International surveys needs to be mindful of the requirement for the cross-cultural equivalence of instruments [64]. In order to address this, we consulted with academics who comprise the Asia-Pacific Scientific Steering Group on Social Quality (PR Ward is a founding member of this group)

Table 1 Survey question 'On the last occasion you needed to see a doctor or medical specialist, to what extent did each of the following factors make it difficult for you to do so?'

\begin{tabular}{lllll}
\hline & $\begin{array}{l}\text { Very } \\
\text { difficult }\end{array}$ & $\begin{array}{l}\text { A little } \\
\text { difficult }\end{array}$ & $\begin{array}{l}\text { Not } \\
\text { difficult } \\
\text { at all }\end{array}$ & $\begin{array}{l}\text { Not applicable / never } \\
\text { needed to see doctor }\end{array}$ \\
\hline $\begin{array}{l}\text { Distance to doctor's office / } \\
\text { hospital / medical centre }\end{array}$ & $\square$ & $\square$ & $\square$ & $\square$ \\
$\begin{array}{l}\text { Delay in getting appointment } \\
\text { Waiting time to see doctor on }\end{array}$ & $\square$ & $\square$ & $\square$ & $\square$ \\
$\begin{array}{l}\text { day of appointment } \\
\text { Cost of seeing the doctor }\end{array}$ & $\square$ & $\square$ & & $\square$ \\
\hline
\end{tabular}


[2]. It was decided among committee members that options provided in the Australian survey were 'very difficult', 'difficult', or 'not difficult at all'. Based on the recommendations of the committee member in Asia, response options were: 'very difficult,' 'difficult,' 'easy', and 'very easy'. Additionally, it was decided that the wording for one independent variable, subjective health satisfaction, differed between the Australian survey and all other countries' surveys; Australia's item options were 'happy', 'average', and 'unhappy' and all other countries' were 'satisfied,' 'neither satisfied nor dissatisfied', and 'unsatisfied'.

Robone et al. (2011) also identify difficulties in using scale variables in cross-country comparisons because there is potential that when faced with the instrument individuals are likely to interpret the meaning of the available response categories in a way that systematically differs across populations or population subgroups [65] which compromises the comparability of the data in cross-country analyses. We recognise that by applying country level estimates within the analysis (mixed effects logistic regression models [66]), we can draw more insightful conclusions for comparing the data. However, this form of analysis in not in line with the aim of this manuscript which is to report on within country results rather than across country comparisons.

Table 2 summarizes the methods employed for the collection of data within Australia, Japan, South Korea, Hong Kong, Taiwan (Taipei), and Thailand. It outlines the methodological issues which are discussed in more detail as limitations in the conclusion of the paper.

Appropriate approvals were obtained within each country to undertake the individual surveys. The authors were granted ethics approval from Flinders University Social and Behavioural Research Ethics Committee to obtain and use the collected data for secondary analysis (project number 5221).

\section{Results}

The reports below are inclusive of only statistics regarding the predictor variables. Table 3 summarizes all of the predictors found to be significant for each of the four dependent variables.

Statistically significant outputs for all binomial regression models for all predictor variables are presented below. Within the following section, any odds ratios of less than one should be interpreted as relating to poor access for the particular group within which we are referring.

\section{Distance}

Table 4 provides statistical results from binomial logistic regression models computed for the dependent variable distance.

\section{Monthly/annual income}

In Australia, respondents who fell in the highest annual income quartile were $45 \%$ more likely that those in the lowest income quartile [OR=0.55, $\mathrm{CI}=0.30-1.00, \mathrm{p}<0.05$ ] and second-lowest income quartile $[\mathrm{OR}=0.54, \mathrm{CI}=0.32-$ $0.91, \mathrm{p}<0.05]$ to state that distance was not a difficulty factor.

In Hong Kong, respondents who fell in the highest annual income quartile were about $75 \%$ more likely to state that distance was not a difficulty factor than those from the lowest income quartile $[\mathrm{OR}=0.27, \mathrm{CI}=0.14-0.52$, $\mathrm{p}<0.001]$.

Table 2 Comparison of survey methods for six countries

\begin{tabular}{|c|c|c|c|c|c|c|}
\hline & Australia & Japan & South Korea & Hong Kong & Taiwan-Taipei & Thailand \\
\hline \multirow[t]{2}{*}{ Fieldwork dates } & Sept. 2009 & 11 Sept. 21 Sept. & 20 Oct. $\sim 10$ & October $2009 \sim$ & 6 Oct. $\sim 16$ & 20 Oct. $\sim 10$ Nov., \\
\hline & & 2009 & Nov., 2009 & October 2010 & Nov., 2009 & 2009 \\
\hline \multirow[t]{4}{*}{ Population } & 18 years old and & 19 years old and & 18 years old and & 18 years old or & Taipei legal & 18 years old and \\
\hline & over & over & over & above & citizens who & over \\
\hline & & & & & are 20 years & \\
\hline & & & & & old or older & \\
\hline Geographic & Nationwide & Nationwide & Nationwide & Hong Kong & Taipei & Nationwide \\
\hline \multicolumn{7}{|l|}{ coverage } \\
\hline \multirow[t]{3}{*}{ Sampling method } & Stratified according & Regionally stratified & Multi - Stages & Quota sampling & Central & Multi - Stages \\
\hline & to state population & proportional & Sampling & based on the & Location Quota & Sampling \\
\hline & & random sampling & & three age ranges & Sampling & \\
\hline Fieldwork & Self-administered & Face to face & Face to face & Telephone & Face to face & Face to face \\
\hline Methods & postal survey & interview & interview & interview & interview & interview \\
\hline Sample size & 1,044 & 1,006 & 1,200 & 681 & 1,200 & 1,200 \\
\hline
\end{tabular}


Table 3 Summary of variables associated with difficulties in access to healthcare

\begin{tabular}{|c|c|c|c|c|c|}
\hline \multicolumn{2}{|l|}{ Country } & $\begin{array}{l}\text { Difficulties due } \\
\text { to distance }\end{array}$ & $\begin{array}{l}\text { Difficulties } \\
\text { in getting } \\
\text { an appointment }\end{array}$ & $\begin{array}{l}\text { Difficulty } \\
\text { due to } \\
\text { waiting times }\end{array}$ & $\begin{array}{l}\text { Difficulties due } \\
\text { to cost }\end{array}$ \\
\hline \multirow[t]{9}{*}{ Australia } & Demographic & Married, divorced, widowed or & Female; married & $\begin{array}{l}\text { Female; worked } \\
\text { part- }\end{array}$ & Female; married or \\
\hline & & separated & & time & cohabiting \\
\hline & Health & Felt very unhappy with their health; & Nil & $\mathrm{Nil}$ & $\mathrm{Nil}$ \\
\hline & related & & & & \\
\hline & Economic & S some savings and borrowed & Just got by, spent & $\begin{array}{l}\text { Just got by } \\
\text { financially }\end{array}$ & Second-lowest income \\
\hline & & money; or fell in the lowest income & savings, or spent & $\begin{array}{l}\text { in the last year, } \\
\text { spent }\end{array}$ & quartile; just got by \\
\hline & & quartile group & $\begin{array}{l}\text { savings and } \\
\text { borrowed }\end{array}$ & $\begin{array}{l}\text { some savings or } \\
\text { spent }\end{array}$ & financially or spend \\
\hline & & & money & some savings and & savings and borrowed \\
\hline & & & & borrowed money & money \\
\hline \multirow[t]{6}{*}{$\begin{array}{l}\text { Hong } \\
\text { Kong }\end{array}$} & Demographic & $\mathrm{Nil}$ & $\begin{array}{l}\text { Aged } 60 \text { years or } \\
\text { older }\end{array}$ & Retired or pensioner & Retired/pensioner \\
\hline & Health & $\mathrm{Nil}$ & $\mathrm{Nil}$ & $\mathrm{Nil}$ & Perceived health to be \\
\hline & related & & & & neither important nor \\
\hline & & & & & unimportant \\
\hline & Economic & $\begin{array}{l}\text { Spent some savings or fell in the } \\
\text { lowest }\end{array}$ & $\mathrm{Nil}$ & Nil & Nil \\
\hline & & income quartile group & & & \\
\hline \multirow[t]{4}{*}{ Japan } & Demographic & Nil & Nil & 30-39 years of age & Nil \\
\hline & Health & Rated their health as very bad & Satisfied with their & Rated their health as & Rated their health as fair, or \\
\hline & related & & health & $\begin{array}{l}\text { good, fair, bad, or } \\
\text { very bad }\end{array}$ & rated their health as bad \\
\hline & Economic & $\mathrm{Nil}$ & $\mathrm{Nil}$ & Nil & $\begin{array}{l}\text { Spent some savings or spent } \\
\text { some savings and borrowed money }\end{array}$ \\
\hline \multirow[t]{5}{*}{ Korea } & Demographic & $\mathrm{Nil}$ & Nil & Nil & Female \\
\hline & Health & Fair state of health, very bad health; & $\mathrm{Nil}$ & $\mathrm{Nil}$ & $\mathrm{Nil}$ \\
\hline & related & chronic health condition & & & \\
\hline & Economic & $\mathrm{Nil}$ & Nil & Nil & Spent savings and borrow \\
\hline & & & & & money \\
\hline \multirow[t]{6}{*}{ Taiwan } & Demographic & Divorced; part-time workers & Nil & Nil & Work part-time \\
\hline & Health & Nil & $\mathrm{Nil}$ & $\mathrm{Nil}$ & $\mathrm{Nil}$ \\
\hline & related & & & & \\
\hline & Economic & Spent their savings or spent their & $\mathrm{Nil}$ & Spend some savings & Just got by, spent savings, \\
\hline & & savings and borrowed & & & spent savings and \\
\hline & & & & & borrowed money \\
\hline \multirow[t]{5}{*}{ Thailand } & & Work for their family, a & Work for their family, & Work for family & $\mathrm{Nil}$ \\
\hline & & housewife/househusband or a & work part-time & & \\
\hline & & student/unemployed/other & & & \\
\hline & & $\begin{array}{l}\text { Rated their health as being good, fair } \\
\text { or }\end{array}$ & Nil & $\mathrm{Nil}$ & $\mathrm{Nil}$ \\
\hline & & bad & & & \\
\hline
\end{tabular}


Table 3 Summary of variables associated with difficulties in access to healthcare (Continued)

\begin{tabular}{|c|c|c|c|}
\hline Just got by financially, spent some & Nil & Just got by & Spend savings, or spenc \\
\hline savings or spent some savings and & & financially, or spent & savings and borrowed \\
\hline borrowed money; and & & their savings and & money \\
\hline
\end{tabular}

\section{Subjective health satisfaction}

In Australia, respondents who felt indifferent $[\mathrm{OR}=3.15$, $\mathrm{CI}=1.74-5.69, \mathrm{p}<0.001]$ or happy $[\mathrm{OR}=3.91, \mathrm{CI}=2.28-$ $6.71, \mathrm{p}<0.001$ ] about the state of their health were three or four times (respectively) more likely to indicate that distance was not a difficulty factor than those who stated they were very unhappy with their health.

Those who stated average satisfaction with their health in Japan $[\mathrm{OR}=2.18, \mathrm{CI}=1.41-3.37, \mathrm{p}<0.001]$ or said they were satisfied $[\mathrm{OR}=1.72, \mathrm{CI}=1.09-2.72, \mathrm{p}<0.05]$ were approximately twice as likely to state that distance was not difficult than those who indicated they were dissatisfied with their health.

In Thailand, respondents who stated they were satisfied with their health were about 1.9 times more likely $[\mathrm{OR}=1.85, \mathrm{CI}=1.15-2.98, \mathrm{p}<0.05]$ to state that distance was not a difficulty than those who stated they were dissatisfied with their health.

\section{Financial situation}

In Australia, respondents who stated they had saved money were about $65 \%$ more likely to state that distance was not a difficulty factor than those who stated they had spent savings and borrowed money $[\mathrm{OR}=0.35$, $\mathrm{CI}=0.19-0.63, \mathrm{p}<0.01]$.

Hong Kong respondents who stated they had saved money in the last year were about $65 \%$ more likely to state that distance was not a difficulty factor than those who stated they had spent some savings $[\mathrm{OR}=0.37$, $\mathrm{CI}=0.20-0.66, \mathrm{p}<0.01]$.

In Taiwan, respondents who stated they had saved money in the last year were about $50 \%$ more likely to state that distance was not a difficulty factor than those respondents who stated they had spent some savings $[\mathrm{OR}=0.51, \mathrm{CI}=0.29-0.88, \mathrm{p}<0.05]$ and respondents who stated they had spent savings and borrowed money [OR=0.49, CI=0.26-0.91, $\mathrm{p}<0.05]$.

Compared with respondents who stated they had spent some savings in Thailand [OR $=0.47, \mathrm{CI}=0.27-0.82$, $\mathrm{p}<0.01$ ] and respondents who selected the item 'just got by' $[\mathrm{OR}=0.49, \mathrm{CI}=0.35-0.70, \mathrm{p}<0.001]$, those who stated they had saved money in the last year in Thailand were about 50\% more like to respond that distance was not a difficulty factor. Additionally, those who stated they had saved money in the last year were about $60 \%$ more likely than those who stated they had spent savings and borrowed money [OR=0.43, $\mathrm{CI}=0.27-0.67, \mathrm{p}<0.001]$ to state that distance was not a difficulty factor.

\section{Self-rated health (Not applicable to Hong Kong \& Taiwan)}

In Japan, respondents who rated their health as being very good were approximately $70 \%$ more likely to state that distance was not a difficulty factor than respondents who rated their health condition as bad [OR $=0.31$, $\mathrm{CI}=0.14-0.68, \mathrm{p}<0.01]$.

Korean respondents who rated their health as very good were about $60 \%$ more likely to state 'not difficult' than those who felt that their health condition was 'fair' $[\mathrm{OR}=0.43, \mathrm{CI}=0.28-0.68, \mathrm{p}<0.001]$. Those who rated their health as being very good were about $75 \%$ more likely to state that distance was not a difficulty factor than those who rated their health as being very bad [OR=0.25, CI=0.09-0.71, $\mathrm{p}<0.01]$.

In Thailand, those who rated their health as being very good were approximately $50 \%$ more likely to state that distance was not a difficulty factor compared with respondents who rated their health condition as good, fair, or bad $[\mathrm{OR}=0.54, \mathrm{CI}=0.35-0.82, \mathrm{p}<0.01 ; \quad \mathrm{OR}=0.47$, $\mathrm{CI}=0.30-0.75, \quad \mathrm{p}<0.01 ; \quad$ and $\mathrm{OR}=0.44, \quad \mathrm{CI}=0.21-0.90$, $\mathrm{p}<0.01$, respectively].

\section{Chronic health condition (Not applicable to Hong Kong, Japan \& Taiwan)}

In Korea respondents who had no chronic health condition were nearly 3.5 times more likely $[\mathrm{OR}=3.34$, $\mathrm{CI}=1.37-8.18, \mathrm{p}<0.01]$ to state not difficult for distance than those who stated they had a chronic health condition.

\section{Marital status}

In Australia, those who stated they had never married were around $70-80 \%$ more likely to report distance was not a difficulty factor in seeing a doctor or a specialist on the last occasion they required compared with those who stated they were married $[\mathrm{OR}=0.26, \mathrm{CI}=0.13-0.52$, $\mathrm{p}<0.001]$, divorced $[\mathrm{OR}=0.19, \mathrm{CI}=0.09-0.45, \mathrm{p}<0.001]$ widowed $[\mathrm{OR}=0.21, \mathrm{CI}=0.08-0.52, \mathrm{p}<0.01]$ or separated [OR=0.22, CI=0.07-0.70, $\mathrm{p}<0.01]$.

In Taiwan, those who had never married were about $70 \%$ more likely than those who stated they were divorced $[\mathrm{OR}=0.34, \mathrm{CI}=0.17-0.69, \mathrm{p}<0.01]$ to state that it was not difficult in terms of distance to travel on the last occasion they required to see a doctor/specialist. 
Table 4 Six binomial logistic regression models for dependent variable: distance

\begin{tabular}{|c|c|c|c|c|c|c|}
\hline \multicolumn{6}{|l|}{ (Models for DV1) } & 6. TL \\
\hline Model fit $x^{2}(\mathrm{df})$ & $110.68(15)^{* *}$ & $55.69(6)^{* * *}$ & $52.00(8)^{* * * *}$ & $39.94(5)^{* * *}$ & $34.96(14)^{* *}$ & $78.43(13)^{* * *}$ \\
\hline $\mathrm{N}$ response/N total & $1029 / 1044$ & $641 / 674$ (95.1\%) & 996/1000 (99.6\%) & 955/1006 (94.9\%) & $1089 / 1200$ (90.8\%) & $1185 / 1200(98.8 \%)$ \\
\hline Nagelkerke $\mathrm{R}^{2}$ & 0.28 & 0.16 & 0.08 & 0.07 & 0.05 & 0.10 \\
\hline \multirow[t]{3}{*}{ Independent Variable } & Wald $x^{2}(d f) p^{*}$ & Waldx $x^{2}(d f) p^{*}$ & Waldx $\left.x^{2} d f\right) p^{*}$ & Wald $x^{2}(d f) p^{*}$ & Wald $x^{2}(d f) p^{*}$ & Wald $x^{2}(d f) p^{*}$ \\
\hline & OR $(95 \% C l)$ & OR $(95 \% \mathrm{Cl})$ & OR $(95 \% C l)$ & OR $(95 \% C l)$ & OR $(95 \% C l)$ & OR $(95 \% C l)$ \\
\hline & Coefficient $b$ & Coefficient $b$ & Coefficient $b$ & Coefficient & Coefficient b & Coefficient b \\
\hline Marital status & $21.35(5)^{* *}$ & $"$ & ' & ' & $11.17 x^{2}(5)^{*}$ & \\
\hline \multirow[t]{2}{*}{ Never married (ref) } & OR 1.00 & & & & OR 1.00 & \\
\hline & 0 & & & & 0 & \\
\hline \multirow[t]{3}{*}{ Married } & $14.69 x^{2}(1)^{* * *}$ & ' & ' & ' & ' & ' \\
\hline & $0.26(0.13-0.52)$ & & & & & \\
\hline & -1.34 & & & & & \\
\hline \multirow[t]{3}{*}{ Divorced } & ' & ' & ' & , & $8.83 x^{2}(1)^{* *}$ & , \\
\hline & & & & & $0.34(0.17-0.69)$ & \\
\hline & & & & & -1.07 & \\
\hline \multirow[t]{3}{*}{ Widowed } & $11.46 x^{2}(1)^{* *}$ & ' & ' & ' & & ' \\
\hline & $0.21(0.08-0.52)$ & & & & & \\
\hline & -1.57 & & & & & \\
\hline \multirow[t]{3}{*}{ Separated } & $6.75 x^{2}(1)^{* *}$ & ' & ' & ' & & , \\
\hline & $0.22(0.07-0.70)$ & & & & & \\
\hline & -1.50 & & & & & \\
\hline Cohabiting & & ' & ' & ' & & ' \\
\hline \multirow[t]{2}{*}{ Work status } & ' & ' & ' & ' & $13.73 x^{2}(6)^{*}$ & $24.24 x^{2}(6)^{* * *}$ \\
\hline & & & & & 1.00 & 1.00 \\
\hline Full-time (ref) & & & & & 0 & 0 \\
\hline \multirow[t]{3}{*}{ Part-time } & ' & ' & ' & ' & $5.76 x^{2}(1)^{*}$ & ' \\
\hline & & & & & $0.50(0.28-0.88)$ & \\
\hline & & & & & -0.70 & \\
\hline Self-employed & ' & ' & ' & ' & $'$ & . \\
\hline \multirow[t]{3}{*}{ Work for family } & ' & ' & ' & ' & ' & $12.92 x^{2}(1)^{* * *}$ \\
\hline & & & & & & $0.47(0.31-0.71)$ \\
\hline & & & & & & -0.76 \\
\hline Retired/ & ' & ' & ' & ' & $4.56 x^{2}(1)^{*}$ & ' \\
\hline \multirow[t]{2}{*}{ Pensioner } & & & & & $2.00(1.06-3.77)$ & \\
\hline & & & & & 0.69 & \\
\hline Housewife/ & ' & ' & ' & ' & ' & $10.24 x^{2}(1)^{* *}$ \\
\hline \multirow[t]{2}{*}{ Househusband } & & & & & & $0.42(0.25-0.72)$ \\
\hline & & & & & & -0.86 \\
\hline Student/ & ' & ' & ' & ' & ' & $6.42 x^{2}(1)^{*}$ \\
\hline Unemployed/ & & & & & & $0.59(0.40-0.89)$ \\
\hline Other & & & & & & -0.54 \\
\hline
\end{tabular}

Subjective health

Satisfaction
$24.67 x^{2}(2)^{* * * *}$

1.00
Dissatisfied (ref)

$12.50 x^{2}(2)^{* *}$
Dissatisfied (ref)

$7.54 x^{2}(2)^{*}$ 
Table 4 Six binomial logistic regression models for dependent variable: distance (Continued)

\begin{tabular}{|c|c|c|c|c|c|c|}
\hline \multirow[t]{2}{*}{ Unhappy (ref) } & \multirow[t]{2}{*}{0} & & \multicolumn{3}{|l|}{1.00} & 1.00 \\
\hline & & & 0 & & & 0 \\
\hline \multirow[t]{4}{*}{ Average } & $14.36 x^{2}(1)^{* * *}$ & , & Average & , & ' & ' \\
\hline & $3.15(1.74-5.69)$ & & $12.34 x^{2}(1)^{* * *}$ & & & \\
\hline & 1.15 & & $2.18(1.41-3.37)$ & & & \\
\hline & & & 0.54 & & & \\
\hline \multirow[t]{4}{*}{ Happy } & $24.52 x^{2}(1)^{* * *}$ & ' & Satisfied & ' & ' & Satisfied \\
\hline & $3.91(2.28-6.71)$ & & $5.32 x^{2}(1)^{*}$ & & & $6.35 x^{2}(1)^{*}$ \\
\hline & 1.36 & & $1.72(1.09-2.72)$ & & & $1.85(1.15-2.98)$ \\
\hline & & & 0.54 & & & 0.61 \\
\hline Financial situation in & $12.88 x^{2}(3)^{* *}$ & $21.52 x^{2}(3)^{* * *}$ & ' & ' & $8.71 x^{2}(3)^{*}$ & $18.71 x^{2}(3)^{* * *}$ \\
\hline last year & 1.00 & 1.00 & & & 1.00 & 1.00 \\
\hline Save money (ref) & 0 & 0 & & & 0 & 0 \\
\hline \multirow[t]{3}{*}{ Just get by } & ' & ' & ' & ' & ' & $15.38 x^{2}(1)^{* * *}$ \\
\hline & & & & & & $0.49(0.35-0.70)$ \\
\hline & & & & & & -0.71 \\
\hline \multirow[t]{3}{*}{ Spent some savings } & ' & $11.11 x^{2}(1)^{* *}$ & ' & ' & $5.82 x^{2}(1)^{*}$ & $7.72 x^{2}(1)^{* *}$ \\
\hline & & $0.37(0.20-0.66)$ & & & $0.51(0.29-0.88)$ & $0.47(0.27-0.82)$ \\
\hline & & -1.00 & & & -0.68 & -0.70 \\
\hline Spent savings \& & $11.87 x^{2}(1)^{* *}$ & , & , & , & $5.16 x^{2}(1)^{*}$ & $13.80 x^{2}(1)^{* * *}$ \\
\hline \multirow[t]{2}{*}{ borrowed money } & $0.35(0.19-0.63)$ & & & & $0.49(0.26-0.91)$ & $0.43(0.27-0.67)$ \\
\hline & -1.06 & & & & -0.72 & -0.85 \\
\hline Household annual or & (annual) & (monthly) & ' & ' & ' & ' \\
\hline monthly adjusted & $9.04 x^{2}(3)^{*}$ & $16.18 x^{2}(3)^{* *}$ & & & & \\
\hline income quartile & 1.00 & 1.00 & & & & \\
\hline $4^{\text {th }}$ : quartile (ref) & 0 & 0 & & & & \\
\hline \multirow[t]{3}{*}{$1^{\text {st }}$ quartile } & $3.83 x^{2}(1)^{*}$ & $14.99 x^{2}(1)^{* * *}$ & ' & ' & ' & ' \\
\hline & $0.55(0.30-1.02)$ & $0.27(0.14-0.52)$ & & & & \\
\hline & -0.59 & -1.32 & & & & \\
\hline \multirow[t]{3}{*}{$2^{\text {nd }}$ quartile } & $5.31 x^{2}(1)^{*}$ & ' & ' & ' & ' & ' \\
\hline & $0.54(0.32-0.93)$ & & & & & \\
\hline & -0.62 & & & & & \\
\hline $3^{\text {rd }}$ : quartile & ' & ' & ' & ' & ' & ' \\
\hline Self-rated health & ' & ' & $14.76 x^{2}(4)^{* *}$ & $22.13 x^{2}(4)^{* * *}$ & ' & $11.47 x^{2}(4)^{*}$ \\
\hline \multirow[t]{2}{*}{ Very good (ref) } & & & 1.00 & 1.00 & & 1.00 \\
\hline & & & 0 & 0 & & 0 \\
\hline \multirow[t]{3}{*}{ Good } & ' & ' & ' & ' & & $8.16 x^{2}(1)^{* *}$ \\
\hline & & & & & & $0.54(0.35-0.82)$ \\
\hline & & & & & & -0.63 \\
\hline \multirow[t]{3}{*}{ Fair } & ' & ' & ' & $13.41 x^{2}(1)^{* * *}$ & ' & $10.29 x^{2}(1)^{* *}$ \\
\hline & & & & $0.43(0.28-0.68$ & & $0.47(0.30-0.75)$ \\
\hline & & & & -0.84 & & -0.76 \\
\hline \multirow[t]{3}{*}{ Bad } & ' & ' & $8.31 x^{2}(1)^{* *}$ & ' & ' & $4.99 x^{2}(1)^{*}$ \\
\hline & & & $0.31(0.14-0.86)$ & & & $0.44(0.21-0.90)$ \\
\hline & & & -1.19 & & & -0.83 \\
\hline
\end{tabular}


Table 4 Six binomial logistic regression models for dependent variable: distance (Continued)

\begin{tabular}{lll}
\hline Very bad & $6.79 x^{2}(1)^{* *}$ \\
& $0.25(0.09-0.71)$ \\
& -1.38 \\
Whether respondent has ' & - \\
chronic health &, & 1.00 \\
condition &, & 0 \\
Yes (ref) &, & $6.99(1)^{* *}$, \\
No & $3.34(1.37-8.18)$ \\
& 1.21 \\
\hline
\end{tabular}

Ref: reference level, ${ }^{*} \mathrm{p}<.05 ;{ }^{* *} \mathrm{p}<.01,{ }^{* *} \mathrm{p}<.001$.

\section{Work status}

In Taiwan, respondents who worked full-time were approximately $50 \%$ more likely to state 'not difficult' in terms of distance than those who worked part-time $[\mathrm{OR}=0.50, \mathrm{CI}=0.28-0.88, \mathrm{p}<0.05]$. Those who were retired/pensioners $[\mathrm{OR}=2.00, \mathrm{CI}=1.06-3.77, \mathrm{p}<0.05]$ were twice as likely as those who worked full-time to state that distance was not a difficulty factor the last time they required to see a doctor or specialist.

In Thailand, respondents who worked full-time were about 55\% more likely to state that distance was not a difficulty factor than those who indicated that they worked for their family $[\mathrm{OR}=0.47, \mathrm{CI}=0.31-0.71, \mathrm{p}<0.001]$. Respondents who worked full-time were found to be nearly $60 \%$ more likely than respondents who were housewives/ househusbands were $[\mathrm{OR}=0.42, \mathrm{CI}=0.25-0.72, \mathrm{p}<0.01]$ and about $40 \%$ more likely than those who indicated stu$\mathrm{dent} /$ unemployed/other $[\mathrm{OR}=0.59, \mathrm{CI}=0.40-0.89, \mathrm{p}<0.05]$ to find distance not a difficulty.

\section{Appointment}

Table 5 provides statistical results for binomial logistic regression models computed for the dependent variable appointment.

\section{Subjective health satisfaction}

Australian respondents who stated they were happy about their health were about 3 times more likely than those who were unhappy about their health $[\mathrm{OR}=3.10$, $\mathrm{CI}=1.73-5.56, \mathrm{p}<0.001]$ and almost 2 times more likely than those who stated that there we 'average' about their health $[\mathrm{OR}=1.78, \mathrm{CI}=0.96-3.29]$ to state that appointment delays were not a difficulty factor.

In Korea, those who stated they were satisfied with their health were nearly twice as likely $[\mathrm{OR}=1.77$, $\mathrm{CI}=1.16-2.71, \mathrm{p}<0.01]$ to state that appointment delay was not a difficulty factor compared with those who stated they were dissatisfied with their health.
In Taiwan respondents who stated they were satisfied with their health were nearly 2.5 times more likely $[\mathrm{OR}=2.31, \mathrm{CI}=1.52-3.51, \mathrm{p}<0.001]$ to state that appointment delay was not a difficulty factor than those who stated they were dissatisfied with their health.

\section{Financial situation in last year}

In Australia those who stated they had saved money were about $45 \%$ more likely to state that appointment delay was not a difficulty compared with those who 'just got by' $[\mathrm{OR}=0.56, \mathrm{CI}=0.39-0.80, \mathrm{p}<0.01]$. Similarly, respondents who stated they had saved money were about $40 \%$ more likely to state that appointment delay was not a difficulty than those who had 'spent some savings' $[\mathrm{OR}=0.62, \mathrm{CI}=0.40-0.94, \mathrm{p}<0.05]$, and about $50 \%$ more likely to state that appointment delay was not a difficulty than those who had 'spent savings and borrowed money' were $[\mathrm{OR}=0.50, \mathrm{CI}=0.28-0.88, \mathrm{p}<0.05]$.

\section{Self-rated health}

In Japan, those who stated they were very dissatisfied with their health were approximately $70 \%$ more likely to state that appointment delay was not a difficulty factor than those who stated they were satisfied with their health $[\mathrm{OR}=0.31, \mathrm{CI}=0.15-0.64 . \mathrm{p}<0.01]$.

\section{Chronic health condition and appointment difficulties}

In Australia respondents who indicated they had no chronic health condition were nearly 1.5 times more likely $[\mathrm{OR}=1.41, \mathrm{CI}=1.00-1.98, \mathrm{p}<0.05]$ to state that appointment delays were not a difficulty than those who indicated having a chronic health condition.

Sex

Within Australia, males were about 50\% more likely to state that it was not difficult to make an appointment the last time they required to see a doctor/GP than females $[\mathrm{OR}=0.50, \mathrm{CI}=0.37-0.69, \mathrm{p}<0.001]$. 
Table 5 Six binomial logistic regression models for dependent variable: appointment

\begin{tabular}{|c|c|c|c|c|c|c|}
\hline \multicolumn{6}{|l|}{ (Models for DV2) } & 6. TL \\
\hline Model fit $x^{2}(d f)$ & $125.79(18)^{* * *}$ & $103.79(5)^{* * *}$ & $36.87(8)^{* * *}$ & $18.45(4)^{* *}$ & $16.50(2)^{* * *}$ & $47.09(11)^{* * *}$ \\
\hline $\mathrm{N}$ response/N total & $816 / 1044(78.2 \%)$ & 667/674 (99.0\%) & $996 / 1000$ (99.6\%) & 990/1006 (98.4\%) & $1157 / 1200(96.4 \%)$ & $1185 / 1200(98.8 \%)$ \\
\hline Nagelkerke $\mathrm{R}^{2}$ & 0.19 & 0.19 & 0.06 & 0.03 & 0.02 & 0.05 \\
\hline \multirow[t]{3}{*}{ Independent Variable } & Wald $x^{2}(d f) p^{*}$ & Wald $x^{2}(d f) p^{*}$ & Waldx $x^{2}(d f) p^{*}$ & Waldx ${ }^{2} d f$ p $p^{*}$ & Wald $x^{2}(d f) p^{*}$ & Waldx ${ }^{2}(d f) p^{*}$ \\
\hline & OR $(95 \% \mathrm{Cl})$ & OR (95\%Cl) & OR $(95 \% \mathrm{Cl})$ & OR $(95 \% \mathrm{Cl})$ & OR $(95 \% \mathrm{Cl})$ & OR $(95 \% \mathrm{Cl})$ \\
\hline & Coefficient & Coefficient & Coefficient & Coefficient & Coefficient & Coefficient \\
\hline
\end{tabular}

Sex

Male (ref)

1.00

0

Female

$18.59 x^{2}(1)^{* * *}$

$00.50(0.37-0.69)$

$-0.69$

Age

$27.55 x^{2}(5)^{* * *}$

$82.60 x^{2}(5)^{* * *}$

$15.27(5)^{* *}$

$<20$ (ref)

1.00

1.00

1.00

0

0

20-29 (ref)

$20.94 x^{2}(1)^{* * *}$

$17.45 x^{2}(4)^{* *}$

18.87 (3.69-31.65)

1.00

2.38

0

30-39

$12.02 x^{2}(1)^{* *}$

6.97 (2.33-20.88)

1.94

40-49

$14.59 x^{2}(1)^{* * *}$

$7.91(2.62-23.91)$

2.07

50-59

$16.74 x^{2}(1)^{* * *}$

10.43 (3.39-32.07)

2.35

$60+$

$$
\begin{array}{lll}
20.83 x^{2}(1)^{* * *} & 30.04 x^{2}(1)^{* * *} & 11.62 x^{2}(1)^{* *} \\
13.86(4.48-42.88) & 0.13(0.06-0.27) & 2.37(1.44-3.90) \\
2.63 & -2.04 & 0.86
\end{array}
$$

\section{Marital status}

$15.05 x^{2}(5)^{* *}$

Never married (ref)

1.00

\section{0}

Married

$$
\begin{aligned}
& 12.56 x^{2}(1)^{* *} \\
& 0.62(0.27-0.70) \\
& -0.83
\end{aligned}
$$

Divorced

Widowed

Separate

Cohabiting

Work status

Full-time

$$
\text { , }
$$


Table 5 Six binomial logistic regression models for dependent variable: appointment (Continued)

\begin{tabular}{|c|c|c|c|c|c|c|}
\hline \multirow[t]{3}{*}{ Part-time } & \multirow[t]{3}{*}{ ' } & \multirow[t]{3}{*}{$\bar{\prime}$} & \multirow[t]{3}{*}{ ' } & \multirow[t]{3}{*}{ ' } & \multirow[t]{3}{*}{ ' } & $6.37 x^{2}(1)^{*}$ \\
\hline & & & & & & $0.45(0.24-0.84)$ \\
\hline & & & & & & -0.80 \\
\hline Self-employed & ' & ' & ' & ' & ' & ' \\
\hline \multirow[t]{3}{*}{ Work for family } & \multirow[t]{3}{*}{ ' } & \multirow[t]{3}{*}{ ' } & \multirow[t]{3}{*}{ ' } & \multirow[t]{3}{*}{, } & \multirow[t]{3}{*}{ ' } & $31.24 x^{2}(1)^{* * *}$ \\
\hline & & & & & & $0.34(0.23-0.49)$ \\
\hline & & & & & & -1.09 \\
\hline Retired/pensioner & ' & ' & ' & ' & ' & ' \\
\hline Housewife/househusband & ' & ' & , & , & , & , \\
\hline Student/ & ' & ' & ' & ' & ' & ' \\
\hline \multicolumn{7}{|l|}{ Unemployed/ } \\
\hline \multicolumn{7}{|l|}{ Other } \\
\hline Subjective health & $19.14 x^{2}(2)^{* * *}$ & ' & , & Dissatisfied (ref) & Dissatisfied (ref) & , \\
\hline satisfaction & 1.00 & & & $15.90 x^{2}(2)^{* * *}$ & $16.73 x^{2}(2)^{* * *}$ & \\
\hline \multirow[t]{2}{*}{ Unhappy (ref) } & 0 & & & 1.00 & 1.00 & \\
\hline & & & & 0 & 0 & \\
\hline \multirow[t]{3}{*}{ Average } & $3.34 x^{2}(1)$ & ' & ' & ' & ' & ' \\
\hline & $1.78(0.96-3.29)$ & & & & & \\
\hline & 0.57 & & & & & \\
\hline \multirow[t]{4}{*}{ Happy } & $14.36 x^{2}(1)^{* * *}$ & ' & ' & Satisfied & Satisfied & ' \\
\hline & $3.10(1.73-5.56)$ & & & $7.02 x^{2}(1)^{* *}$ & $15.20 x^{2}(1)^{* * *}$ & \\
\hline & 1.13 & & & $1.77(1.16-2.71)$ & $2.31(1.52-3.51)$ & \\
\hline & & & & 0.57 & 0.84 & \\
\hline Financial situation in & $12.38 x^{2}(3)^{* *}$ & ' & ' & , & ' & , \\
\hline last year & 1.00 & & & & & \\
\hline Save money (ref) & 0 & & & & & \\
\hline \multirow[t]{3}{*}{ Just get by } & $9.85 x^{2}(1)^{* *}$ & ' & ' & ' & ' & ' \\
\hline & $0.56(0.39-0.80)$ & & & & & \\
\hline & -0.59 & & & & & \\
\hline \multirow[t]{3}{*}{ Spent some savings } & $4.97 X^{2}(1)^{*}$ & , & , & , & , & , \\
\hline & $0.62(0.40-0.94)$ & & & & & \\
\hline & -0.49 & & & & & \\
\hline Spent savings \& & $5.70 x^{2}(1)^{*}$ & ' & , & , & ' & , \\
\hline \multirow[t]{2}{*}{ borrowed money } & $0.50(0.28-0.88)$ & & & & & \\
\hline & -0.69 & & & & & \\
\hline Self-rated health & ' & ' & $26.46 x^{2}(4)^{* * *}$ & ' & ' & ' \\
\hline \multirow[t]{2}{*}{ Very dissatisfied } & & & 1.00 & & & \\
\hline & & & 0 & & & \\
\hline Dissatisfied & ' & ' & ' & ' & ' & ' \\
\hline Average & ' & ' & ' & ' & ' & ' \\
\hline \multirow[t]{3}{*}{ Satisfied } & , & ' & $10.30 x^{2}(1)^{* *}$ & , & , & , \\
\hline & & & $0.31(0.15-0.64)$ & & & \\
\hline & & & -1.16 & & & \\
\hline Very satisfied & ' & ' & , & , & ' & , \\
\hline
\end{tabular}


Table 5 Six binomial logistic regression models for dependent variable: appointment (Continued)

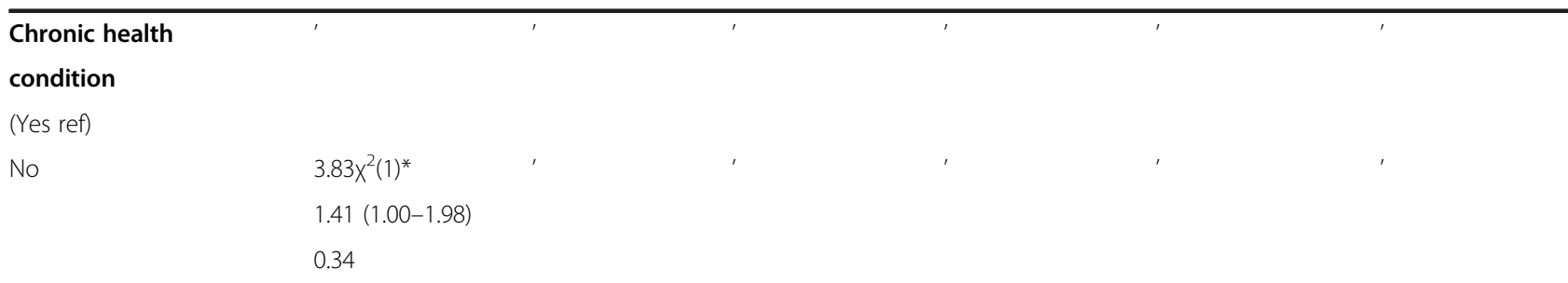

Ref: reference level, ${ }^{*} \mathrm{p}<.05 ;{ }^{* *} \mathrm{p}<.01,{ }^{* * *} \mathrm{p}<.001$.

\section{Age}

Australian respondents in all age groups from 20 years of age and over were at least 6 times more likely to state that making an appointment was not a difficulty factor. For example, those who were aged between 30-39 $[\mathrm{OR}=6.97, \mathrm{CI}=2.33-20.88, \mathrm{p}<0.001]$ were about 7 times more likely to state that appointment was not a difficulty factor, and those who were aged 60 or above were about 14 times more likely to state that appointment making was not a difficulty factor $[\mathrm{OR}=13.86, \mathrm{CI}=4.48-42.89$, $\mathrm{p}<0.001]$.

Age was the only significant predictor of appointment difficulties for Hong Kong. Respondents aged 20 years or younger were about $85 \%$ more likely to indicate that scheduling an appointment was not a difficulty factor on the last occasion they required to see a doctor/specialist than those who were aged 60 years or over $[\mathrm{OR}=0.13$, $\mathrm{CI}=0.06-0.27, \mathrm{p}<0.001]$.

In Japan, those who were 60 years or older were nearly 2.5 times more likely $[\mathrm{OR}=2.37, \mathrm{CI}=1.44=3.90, \mathrm{p}<0.01]$ to state that appointment making was not a difficulty factor on the last occasion they required to see a doctor/specialist compared with those who were aged 20-29 years.

\section{Marital status}

In Australia, respondents who had never been married were about $55 \%$ more likely to state that appointment making delays was not a difficult factor than those who were married $[\mathrm{OR}=0.42, \mathrm{CI}=0.27-0.70, \mathrm{p}<0.01]$.

\section{Work status}

In Thailand, respondents who worked full-time were about $55 \%$ more likely to state that appointment delay was not a difficulty factor the last time they required to see a doctor/specialist than those who worked part-time $[\mathrm{OR}=0.45, \mathrm{CI}=0.24-0.84, \mathrm{p}<0.05]$. Those who were fulltime workers were about $65 \%$ more likely to state that appointment delay was not a difficulty factor than those who worked for their family [OR=0.34, $\mathrm{CI}=0.23-0.49, \mathrm{p}<0.001]$.

\section{Waiting time}

Table 6 provides statistical results from binomial logistic regression models computed for the dependent variable waiting time.

\section{Subjective health satisfaction}

Australian respondents who stated they felt happy about their health were about 3 times more likely [OR $=2.57$, $\mathrm{CI}=1.54-4.28, \mathrm{p}<0.001]$ to state that waiting times are not a difficulty factor.

For Korea, respondents who were satisfied with their health were approximately 1.8 times more likely $[\mathrm{OR}=1.78, \mathrm{CI}=1.15-2.74, \mathrm{p}<0.01]$ to indicate that waiting time was not a difficulty factor than respondents who were dissatisfied with their health.

Taiwan respondents who were satisfied with their health were over 1.5 times more likely $[\mathrm{OR}=1.56$, $\mathrm{CI}=1.06-2.29, \mathrm{p}<0.05]$ to state that waiting time was not a difficulty factor than those who were dissatisfied with their health.

\section{Financial situation in last year}

In Australia, those who stated they had saved money were about $35 \%$ more likely to state that waiting time was not a difficulty compared with those who just got by' $[\mathrm{OR}=0.66, \mathrm{CI}=0.45-0.91, \mathrm{p}<0.05]$. Similarly, those who had saved money were about $60 \%$ more likely than those who had 'spent some savings' $[\mathrm{OR}=0.38, \mathrm{CI}=0.28-$ $0.67, \mathrm{p}<0.05$ ] and $45 \%$ more likely those who 'spent savings and borrowed money' $[\mathrm{OR}=0.56, \mathrm{CI}=0.31-1.01]$ to state that waiting time was not a difficulty.

Taiwan respondents who had saved money were about $45 \%$ more likely than those who had spent some savings to state that waiting time was not a difficulty factor [OR=0.55, CI $=0.36-0.85, \mathrm{p}<0.01]$.

In Thailand, individuals who had saved money were about 35\% more likely to state that waiting time was not a difficulty factor compared with those how 'just got by' [OR=0.66, CI=0.49-0.89, $\mathrm{p}<0.01]$. Similarly, individuals who had saved money were nearly $50 \%$ more likely than respondents who spent savings and borrowed money to state that waiting time was not a difficulty factor $[\mathrm{OR}=0.48, \mathrm{CI}=0.32-0.71, \mathrm{p}<0.001]$.

\section{Self-rated health}

In Japan, those who rated their health as very good were about 30\% more likely than respondents who rated their health as good to indicate waiting time was not a difficulty factor $[\mathrm{OR}=0.72, \mathrm{CI}=0.58-0.89, \mathrm{p}<0.05]$. Again, 
Table 6 Six binomial logistic regression models for dependent variable: waiting time

\begin{tabular}{|c|c|c|c|c|c|c|}
\hline Country (Models for DV3) & 1. AU & 2. HK & 3. JP & 4. $K R$ & 5. TW & 6. TL \\
\hline Model fit $X^{2}(d f)$ & $110.93(21)^{* * *}$ & $108.49(5)^{* * *}$ & $94.06(10)^{* * *}$ & $14.49(4)^{* *}$ & $21.20(5)^{* *}$ & $48.49(14)^{* * *}$ \\
\hline $\mathrm{N}$ response/N total & $814 / 1044(78 \%)$ & $666 / 674(98.8 \%)$ & $994 / 1000(99.4 \%)$ & $986 / 1006(98 \%)$ & $1082 / 1200(90.2 \%)$ & $1178 / 1200(98.2 \%)$ \\
\hline Nagelkerke $\mathrm{R}^{2}$ & 0.17 & 0.20 & 0.12 & 0.02 & 0.03 & 0.05 \\
\hline \multirow[t]{3}{*}{ Independent variable } & Waldx ${ }^{2}(d f) p^{*}$ & Waldx ${ }^{2}(d f) p^{*}$ & Waldx ${ }^{2}(d f) p^{*}$ & Waldx $\left.{ }^{2} d f\right) p^{*}$ & Waldx ${ }^{2}(d f) p^{*}$ & Waldx $x^{2}(d f) p^{*}$ \\
\hline & OR $(95 \% \mathrm{Cl})$ & OR $(95 \% \mathrm{Cl})$ & OR $(95 \% \mathrm{Cl})$ & OR $(95 \% \mathrm{Cl})$ & OR $(95 \% \mathrm{Cl})$ & OR $(95 \% \mathrm{Cl})$ \\
\hline & Coefficient & Coefficient & Coefficient & Coefficient & Coefficient & Coefficient \\
\hline Sex & - & ' & ' & ' & ' & ' \\
\hline \multicolumn{7}{|l|}{ Male (ref) } \\
\hline \multirow[t]{3}{*}{ Female } & $8.11 x^{2}(1)^{* *}$ & ' & , & ' & ' & , \\
\hline & $0.67(0.51-0.88)$ & & & & & \\
\hline & -0.54 & & & & & \\
\hline Age & ' & ' & $20.63 x^{2}(5)^{* *}$ & ' & ' & , \\
\hline \multirow[t]{2}{*}{$<20$ (ref) } & & & 1.00 & & & \\
\hline & & & 0 & & & \\
\hline $20-29$ & ' & ' & ' & ' & ' & , \\
\hline \multirow[t]{3}{*}{$30-39$} & ' & ' & $5.27 x^{2}(1)^{*}$ & ' & ' & , \\
\hline & & & $0.56(0.34-0.92)$ & & & \\
\hline & & & -0.58 & & & \\
\hline $40-49$ & ' & ' & ' & ' & ' & , \\
\hline $50-59$ & ' & ' & ' & ' & ' & ' \\
\hline $60+$ & ' & ' & ' & ' & ' & ' \\
\hline Work status & $40.22 x^{2}(6)^{* *}$ & $68.04 x^{2}(5)^{* * *}$ & ' & ' & ' & $14.01 x^{2}(6)^{*}$ \\
\hline Full-time & 1.00 & 1.00 & & & & 1.00 \\
\hline (ref) & 0 & 0 & & & & 0 \\
\hline \multirow[t]{3}{*}{ Part-time } & $4.82 x^{2}(1)^{*}$ & , & , & ' & ' & , \\
\hline & $0.62(0.41-0.95)$ & & & & & \\
\hline & -0.47 & & & & & \\
\hline Self-employed & ' & ' & ' & ' & ' & ' \\
\hline \multirow[t]{3}{*}{ Working for the family } & ' & , & ' & ' & ' & $11.16 x^{2}(1)^{* *}$ \\
\hline & & & & & & $0.52(0.35-0.76)$ \\
\hline & & & & & & -0.66 \\
\hline \multirow[t]{3}{*}{ Retired/pensioner } & $20.72 x^{2}(1)^{* * *}$ & $66.34 x^{2}(1)^{* * *}$ & ' & ' & ' & ' \\
\hline & $2.45(1.67-3.61)$ & $0.68(0.34-0.13)$ & & & & \\
\hline & 0.90 & -2.69 & & & & \\
\hline Housewife/househusband & ' & ' & ' & ' & ' & ' \\
\hline Student/ & $4.89 x^{2}(1)^{*}$ & , & , & ' & ' & , \\
\hline Unemployed/ & $1.66(1.06-2.60)$ & & & & & \\
\hline Other & 0.51 & & & & & \\
\hline Subjective health & $28.51 x^{2}(2)^{* * *}$ & , & , & Dissatisfied (ref) & Dissatisfied (ref) & , \\
\hline satisfaction & 1.00 & & & $14.41 x^{2}(2)^{* *}$ & $12.68 x^{2}(2)^{* *}$ & \\
\hline \multirow[t]{2}{*}{ Unhappy } & 0 & & & 1.00 & 1.00 & \\
\hline & & & & 0 & 0 & \\
\hline
\end{tabular}


Table 6 Six binomial logistic regression models for dependent variable: waiting time (Continued)

\begin{tabular}{|c|c|c|c|c|c|c|}
\hline Fair & ' & ' & ' & ' & ' & ' \\
\hline \multirow[t]{4}{*}{ Happy } & $13.20 x^{2}(1)^{* * *}$ & ' & ' & Satisfied & Satisfied & ' \\
\hline & $2.57(1.54-4.27)$ & & & $6.79 x^{2}(1)^{* *}$ & $5.02 x^{2}(1)^{*}$ & \\
\hline & 0.94 & & & $1.78(1.15-2.74)$ & $1.56(1.06-2.29)$ & \\
\hline & & & & 0.70 & 0.44 & \\
\hline Financial situation in last & $15.85 x^{2}(3)^{* *}$ & ' & ' & ' & $8.39 x^{2}(3)^{*}$ & $14.74 x^{2}(3)^{* *}$ \\
\hline year & 1.00 & & & & 1.00 & 1.00 \\
\hline Save money (ref) & 0 & & & & 0 & 0 \\
\hline \multirow[t]{3}{*}{ Just get by } & $5.98 x^{2}(1)^{*}$ & ' & ' & ' & ' & $7.65 x^{2}(1)^{* *}$ \\
\hline & $0.66(0.45-0.91)$ & & & & & $0.66(0.49-0.89)$ \\
\hline & -0.45 & & & & & -0.41 \\
\hline \multirow[t]{3}{*}{ Spent some savings } & $14.32 x^{2}(1)^{* * *}$ & ' & ' & ' & $7.26 x^{2}(1)^{* *}$ & , \\
\hline & $0.38(0.28-0.67)$ & & & & $0.55(0.36-0.85)$ & \\
\hline & -0.85 & & & & -0.59 & \\
\hline Spent savings \& borrowed & $3.72 x^{2}(1)^{*}$ & ' & ' & ' & ' & $13.17 x^{2}(1)^{* * *}$ \\
\hline \multirow[t]{2}{*}{ money } & $0.56(0.31-1.01)$ & & & & & $0.48(0.32-0.71)$ \\
\hline & -0.58 & & & & & -0.74 \\
\hline Self-rated health & ' & ' & $44.73 x^{2}(4)^{* * *}$ & , & ' & ' \\
\hline \multirow[t]{2}{*}{ Very good (ref) } & & & 1.00 & & & \\
\hline & & & 0 & & & \\
\hline \multirow[t]{3}{*}{ Good } & ' & ' & $8.95 x^{2}(1)^{* *}$ & ' & ' & ' \\
\hline & & & $0.72(0.58-0.89)$ & & & \\
\hline & & & -0.33 & & & \\
\hline \multirow[t]{3}{*}{ air } & ' & , & $31.63 x^{2}(1)^{* * *}$ & ' & ' & , \\
\hline & & & $0.53(0.43-0.66)$ & & & \\
\hline & & & -0.63 & & & \\
\hline \multirow[t]{3}{*}{$\mathrm{Bad}$} & ' & ' & $27.82 x^{2}(1)^{* * *}$ & ' & ' & , \\
\hline & & & $0.43(0.31-0.59)$ & & & \\
\hline & & & -0.85 & & & \\
\hline \multirow[t]{3}{*}{ Very bad } & ' & ' & $8.34 x^{2}(1)^{* *}$ & ' & ' & ' \\
\hline & & & $0.41(0.23-0.75)$ & & & \\
\hline & & & -0.88 & & & \\
\hline
\end{tabular}

ref: reference level, ${ }^{*} p<.05 ;{ }^{* *} p<.01,{ }^{* * *} p<.001$.

those who rated their health as very good were about $50 \%$ more likely to indicate waiting time was not a difficulty factor than those who rated their health as fair $[\mathrm{OR}=0.53, \mathrm{CI}=0.43-0.66, \mathrm{p}<0.001]$ and approximately $60 \%$ more likely to indicate waiting time was not a difficulty factor than those who rated their health as bad $[\mathrm{OR}=0.43, \quad \mathrm{CI}=0.31-0.59, \quad \mathrm{p}<0.001] \quad$ or $\quad$ very $\quad$ bad $[\mathrm{OR}=0.41, \mathrm{CI}=0.23-0.75, \mathrm{p}<0.01]$.

\section{Sex}

Only for Australia did the final logistic regression model show sex to be a significant indicator of waiting time difficulties, with males about $35 \%$ more likely than Australian females $[\mathrm{OR}=0.67, \mathrm{CI}=0.51-0.88, \mathrm{p}<0.01]$ to state that waiting time was not a difficulty factor on the last occasion they required to see a doctor/specialist.

\section{Age}

In Japan, than those who indicated to be under 20 years of age were about $40 \%$ more likely to state that waiting time was a not difficulty factor on the last occasion they required to see a doctor/specialist than those 30-39 years of age $[\mathrm{OR}=0.56, \mathrm{CI}=0.34-0.92$, $\mathrm{p}<0.05]$. 


\section{Work status}

Respondents who worked full-time were about $40 \%$ more likely to state that waiting time was not a difficulty factor than those who worked part-time $[\mathrm{OR}=0.62$, $\mathrm{CI}=0.41-0.95, \mathrm{p}<0.05]$. Respondents who were retired or pensioners were about 2.5 times more likely $[\mathrm{OR}=2.45$, $\mathrm{CI}=1.67-3.61, \mathrm{p}<0.001]$ to state that waiting time was not a difficulty factor, and respondents who were students/unemployed/other were about 1.5 times $[\mathrm{OR}=1.66$, $\mathrm{CI}=1.06-2.60, \mathrm{p}<0.05]$ more likely to state waiting time was a not difficulty factor compared with those who worked full-time.

In Hong Kong, respondents who worked full-time were about $30 \%$ more likely to state that waiting time was not a difficulty factor on the last occasion they required to see a doctor/specialist than those who stated they were retired/pensioners $[\mathrm{OR}=0.68, \mathrm{CI}=0.34-0.13$, $\mathrm{p}<0.001]$.

In Thailand, respondents who stated they worked fulltime were about $50 \%$ more likely to state that waiting time was not a difficulty factor than those who worked for their family $[\mathrm{OR}=0.52, \mathrm{CI}=0.35-0.76, \mathrm{p}<0.01]$.

\section{Cost}

Table 7 provides statistical results from binomial logistic regression models computed for the dependent variable cost.

\section{Household monthly/annual income}

In Australia those who were in the highest income quartile were approximately $50 \%$ more likely to state cost was not a difficulty factor than respondents who belonged to the second lowest income quartile $[\mathrm{OR}=0.52, \mathrm{CI}=0.33-0.81, \mathrm{p}<0.01]$.

\section{Subjective health satisfaction}

Australian respondents who indicated they were happy with their health were twice as likely $[\mathrm{OR}=2.01, \mathrm{CI}=1.15-$ $3.50, \mathrm{p}<0.05]$ to state that cost was not a difficulty factor than those who were unhappy with their health.

In Hong Kong, individuals who were neither satisfied nor dissatisfied with their health were about 2.5 times more likely $[\mathrm{OR}=2.41, \mathrm{CI}=1.20-5.27, \mathrm{p}<0.05]$ to indicate that cost was not a difficulty factor than those who were dissatisfied with their health. Respondents who were satisfied with their health were about 3.2 times more likely $[\mathrm{OR}=3.24, \mathrm{CI}=1.58-6.62, \mathrm{p}<0.01]$ to state cost was not a difficulty factor than those who were dissatisfied with their health.

Korean respondents who were satisfied with their health were about 2.7 times more likely $[\mathrm{OR}=2.68,1.65-$ $4.33, \mathrm{p}<0.001$ ] to state that cost was not a difficulty factor than those who were dissatisfied with their health.

\section{Financial situation in last year}

Australians who had saved money were about $60 \%$ more likely than those who 'just got by' $[\mathrm{OR}=0.67, \mathrm{CI}=0.45-$ 0.99, $\mathrm{p}<0.05$ ] and about $70 \%$ more than those who had spent savings and borrowed money $[\mathrm{OR}=0.31, \mathrm{CI}=0.18-$ $0.40, \mathrm{p}<0.001]$ to state that cost was not a difficulty factor.

In Japan, those who had saved money in the last year were about $40-50 \%$ more likely to state that cost was not a difficulty factor compared with respondents who stated that in the last year they spent some savings $[\mathrm{OR}=0.53, \mathrm{CI}=0.37-0.76, \mathrm{p}<0.01]$ or spent savings and borrowed money [OR=0.38, $\mathrm{CI}=0.23-0.65, \mathrm{p}<0.001]$.

As for Korean respondents, those who had saved money were about $70 \%$ more likely than those who had spent savings and borrowed money to state that cost was not a difficulty factor $[\mathrm{OR}=0.32, \mathrm{CI}=0.17-0.61$, $\mathrm{p}<0.001]$.

Taiwanese respondents who stated they had saved money in the last year were about $70 \%$ or $75 \%$ respectively more likely to state that cost was not a difficulty factor than those who stated 'just got by' $[\mathrm{OR}=0.32$, $\mathrm{CI}=0.21-0.51, \quad \mathrm{p}<0.001], \quad$ or 'spent some savings' $[\mathrm{OR}=0.25, \mathrm{CI}=0.14-0.44, \mathrm{p}<0.001]$. Those who had saved money were about $85 \%$ more likely to indicate that cost was not a difficulty factor compared with those who had spent savings and borrowed money $[\mathrm{OR}=0.16, \mathrm{CI}=0.09$ $0.30, \mathrm{p}<0.001]$.

In Thailand, respondents who had saved money in the last year were about $50 \%$ or $55 \%$ respectively more likely to state that cost was not a difficulty factor than those who stated 'just got by' $[\mathrm{OR}=0.55, \mathrm{CI}=0.38-0.80$, $\mathrm{p}<0.01]$, or 'spent some savings' $[\mathrm{OR}=0.45, \mathrm{CI}=0.25-$ $0.80, \mathrm{p}<0.01]$. Similarly, those who had saved money were about $55 \%$ more likely those who had spent savings and borrowed money to indicate that cost was not a difficulty factor $[\mathrm{OR}=0.48, \mathrm{CI}=0.30-0.77, \mathrm{p}<0.01]$.

\section{Self-rated health}

In Japan, those who rated their health as very good were about $50 \%$ more likely to indicate cost was not a difficulty factor compared to respondents who rated their health condition as fair $[\mathrm{OR}=0.48, \mathrm{CI}=0.29-0.80$, $\mathrm{p}<0.01]$. Those who rated their health as very good were nearly $75 \%$ more likely to indicate that cost was not a difficulty factor than those who rated their health as bad $[\mathrm{OR}=0.27, \mathrm{CI}=0.14-0.53, \mathrm{p}<0.001]$.

\section{Chronic health condition}

In Korea, respondents who indicated having no chronic health condition were about 2.5 times more likely $[\mathrm{OR}=2.51, \mathrm{CI}=1.25-5.04, \mathrm{p}<0.05]$ to state that cost was not a difficulty factor compared with those who suffered from a chronic health condition. 
Table 7 Six binomial logistic regression models for dependent variable: cost

\begin{tabular}{|c|c|c|c|c|c|c|}
\hline \multicolumn{7}{|l|}{ (Models for DV4) } \\
\hline Model fit $x^{2}(\mathrm{~d} f)$ & $106.91(20)^{* * *}$ & $121.19(13)^{* * *}$ & $99.37(13)^{* * *}$ & $102.49(15)^{* * *}$ & $76.75(9)^{* * *}$ & $29.28(8)^{* * *}$ \\
\hline $\mathrm{N}$ response/N total & 912/1044 (87.4\%) & 665/674 (98.8\%) & $990 / 1000$ (99\%) & $961 / 1006(95.5 \%)$ & 1081/1200 (90.1\%) & $1185 / 1200(98.8 \%)$ \\
\hline Nagelkerke $\mathrm{R}^{2}$ & 0.16 & 0.23 & 0.13 & 0.14 & 0.12 & 0.04 \\
\hline \multirow[t]{3}{*}{ Independent variable } & Wald $x^{2}(d f) p^{*} O R$ & Wald $x^{2}(d f) p^{*} O R$ & Wald $x^{2}(d f) p^{*}$ & Wald $\left.x^{2} d f\right) p^{*}$ & Wald $x^{2}(d f) p^{*}$ & Wald $x^{2}(d f) p^{*}$ \\
\hline & $(95 \% C l)$ & $(95 \% C l)$ & OR $(95 \% C l)$ & OR $(95 \% C l)$ & OR $(95 \% C l)$ & OR $(95 \% C l)$ \\
\hline & Coefficient & Coefficient & Coefficient & Coefficient & Coefficient & Coefficient \\
\hline Sex & - & ' & ' & & ' & ' \\
\hline \multirow[t]{2}{*}{ Male (ref) } & 1.00 & & & 1.00 & & \\
\hline & 0 & & & 0 & & \\
\hline \multirow[t]{3}{*}{ Female } & $12.82 x^{2}(1)^{* * *}$ & ' & ' & $4.58 x^{2}(1)^{*}$ & ' & ' \\
\hline & $0.54(0.38-0.76)$ & & & $0.72(0.54-0.97)$ & & \\
\hline & -0.62 & & & -0.33 & & \\
\hline Age & ' & ' & (20-29 ref) & ' & ' & $15.90 x^{2}(5)^{* *}$ \\
\hline \multirow[t]{2}{*}{$<20$ (ref) } & & & & & & 1.00 \\
\hline & & & & & & 0 \\
\hline \multirow[t]{3}{*}{$20-29$} & ' & ' & $16.72 x^{2}(4)^{* *}$ & , & ' & , \\
\hline & & & 1.00 & & & \\
\hline & & & 0 & & & \\
\hline $30-39$ & ' & ' & ' & ' & ' & ' \\
\hline $40-49$ & ' & ' & ' & ' & ' & ' \\
\hline $50-59$ & $'$ & $'$ & ' & ' & ' & ' \\
\hline \multirow[t]{3}{*}{$60+$} & ' & ' & $8.08 x^{2}(1)^{* *}$ & ' & ' & ' \\
\hline & & & $1.89(1.22-2.94)$ & & & \\
\hline & & & 0.64 & & & \\
\hline Marital status & $15.51(5)^{* *}$ & $32.12(4)^{* * *}$ & ' & $13.09 x^{2}(4)^{* *}$ & ' & ' \\
\hline \multirow[t]{2}{*}{ Never married (ref) } & 1.00 & 1.00 & & 1.00 & & \\
\hline & 0 & 0 & & 0 & & \\
\hline \multirow[t]{3}{*}{ Married } & $9.18(1)^{* *}$ & ' & ' & $8.08 x^{2}(1)^{* *}$ & ' & ' \\
\hline & $0.48(0.30-0.77)$ & & & $0.58(0.40-0.85)$ & & \\
\hline & -0.73 & & & -0.54 & & \\
\hline Divorced & ' & ' & ' & ' & ' & ' \\
\hline \multirow[t]{4}{*}{ Widowed } & ' & $20.12(1)^{* * *}$ & ' & $7.02 x^{2}(1)^{* *}$ & ' & ' \\
\hline & & $19.81(5.37-$ & & $0.38(0.19-0.78)$ & & \\
\hline & & 73.05) & & -0.96 & & \\
\hline & & 2.97 & & & & \\
\hline Separated & $'$ & ' & ' & ' & ' & ' \\
\hline \multirow[t]{3}{*}{ Cohabiting } & $9.23 x^{2}(1)^{* *}$ & ' & ' & ' & ' & ' \\
\hline & $0.38(0.20-0.71)$ & & & & & \\
\hline & -0.98 & & & & & \\
\hline Household monthly & $9.72 x^{2}(3)^{*}$ & ' & ' & ' & ' & ' \\
\hline adjusted income quartile & 1.00 & & & & & \\
\hline $4^{\text {th }}:$ quartile (ref) & 0 & & & & & \\
\hline $1^{\text {st }}$ quartile & ' & ' & ' & ' & ' & , \\
\hline
\end{tabular}


Table 7 Six binomial logistic regression models for dependent variable: cost (Continued)

\begin{tabular}{|c|c|c|c|c|c|c|}
\hline \multirow[t]{3}{*}{$2^{\text {nd }}$ quartile } & $8.11 x^{2}(1)^{* *}$ & ' & , & ' & ' & ' \\
\hline & $0.52(0.33-0.81)$ & & & & & \\
\hline & -0.66 & & & & & \\
\hline $3^{\text {rd }}$ quartile & ' & ' & ' & ' & ' & , \\
\hline Work status & $21.17 x^{2}(6)^{* *}$ & $58.00 x^{2}(5)^{* * *}$ & ' & ' & $21.23 x^{2}(6)^{* *}$ & , \\
\hline Full-time & 1.00 & 1.00 & & & 1.00 & \\
\hline (ref) & 0 & 0 & & & 0 & \\
\hline \multirow[t]{3}{*}{ Part-time } & ' & ' & ' & ' & $18.30 x^{2}(1)^{* * *}$ & , \\
\hline & & & & & $0.31(0.18-0.53)$ & \\
\hline & & & & & -1.18 & \\
\hline \multirow[t]{3}{*}{ Self-employed } & ' & $0.00 x^{2}(1)$ & ' & ' & ' & ' \\
\hline & & - (not given) & & & & \\
\hline & & $20.39^{* * *}$ & & & & \\
\hline Working for the family & ' & ' & ' & ' & ' & ' \\
\hline \multirow[t]{3}{*}{ Retired/pensioner } & $15.92 x^{2}(1)^{* * *}$ & $49-38 x^{2}(1)^{* * *}$ & , & ' & ' & , \\
\hline & $3.32(1.84-5.99)$ & $0.12(0.06-0.21)$ & & & & \\
\hline & 1.20 & -2.15 & & & & \\
\hline \multirow[t]{3}{*}{ Housewife/househusband } & $3.90 x^{2}(1)^{*}$ & , & , & , & ' & , \\
\hline & $2.03(1.01-4.08)$ & & & & & \\
\hline & 0.71 & & & & & \\
\hline Student/ & ' & ' & ' & ' & ' & ' \\
\hline \multicolumn{7}{|l|}{ Unemployed/ } \\
\hline \multicolumn{7}{|l|}{ Other } \\
\hline Subjective health & $6.28 x^{2}(2)^{*}$ & Dissatisfied (ref) & , & Dissatisfied & ' & , \\
\hline satisfaction & 1.00 & $10.65 x^{2}(2)^{* *}$ & & (ref) & & \\
\hline \multirow[t]{3}{*}{ Unhappy } & 0 & 1.00 & & $19.55 x^{2}(2)^{* * *}$ & & \\
\hline & & 0 & & 1.00 & & \\
\hline & & & & 0 & & \\
\hline \multirow[t]{4}{*}{ Fair } & ' & Average & ' & ' & ' & ' \\
\hline & & $5.95 x^{2}(1)^{*}$ & & & & \\
\hline & & $2.41(1.20-5.27)$ & & & & \\
\hline & & 0.92 & & & & \\
\hline \multirow[t]{4}{*}{ Happy } & $5.99 x^{2}(1)^{*}$ & Satisfied & ' & Satisfied & ' & , \\
\hline & $2.01(1.15-3.50)$ & $10.32 x^{2}(1)^{* *}$ & & $16.00 x^{2}(1)^{* * *}$ & & \\
\hline & -0.70 & $3.24(1.58-6.62)$ & & $2.68(1.65-4.33)$ & & \\
\hline & & 1.17 & & 0.98 & & \\
\hline Financial situation in last & $18.19 x^{2}(3)^{* * *}$ & ' & $18.44 x^{2}(3)^{* * *}$ & $12.54 x^{2}(3)^{* *}$ & $41.39 x(3)^{* * *}$ & $13.40 x^{2}(3)^{* *}$ \\
\hline year & 1.00 & & 1.00 & 1.00 & 1.00 & 1.00 \\
\hline Save money (ref) & 0 & & 0 & 0 & 0 & 0 \\
\hline \multirow[t]{3}{*}{ Just get by } & $2.01 x^{2}(1)^{*}$ & ' & ' & , & $24.47 x^{2}(1)^{* * *}$ & $9.98 x^{2}\left(10^{* *}\right)$ \\
\hline & $0.67(0.45-0.99)$ & & & & $0.32(0.21-0.51)$ & $0.55(0.38-0.80)$ \\
\hline & -0.40 & & & & -1.13 & -0.61 \\
\hline \multirow[t]{3}{*}{ Spent some savings } & ' & ' & $11.76 x^{2}(1)^{* *}$ & ' & $22.44 x^{2}(1)^{* * *}$ & $7.46 x^{2}(1)^{* *}$ \\
\hline & & & $0.53(0.37-0.76)$ & & $0.25(0.14-0.44)$ & $0.45(0.25-0.80)$ \\
\hline & & & -0.64 & & -1.41 & -0.80 \\
\hline
\end{tabular}


Table 7 Six binomial logistic regression models for dependent variable: cost (Continued)

\begin{tabular}{|c|c|c|c|c|c|c|}
\hline Spent savings \& borrowed & $17.73 x^{2}(1)^{* * *}$ & ' & $12.50 x^{2}(1)^{* * *}$ & $12.13 x^{2}(1)^{* * *}$ & $33.38 x^{2}(1)^{* * *}$ & $9.22 x^{2}(1)^{* *}$ \\
\hline \multirow[t]{2}{*}{ money } & \multicolumn{2}{|l|}{$0.31(0.18-0.4)$} & $0.38(0.23-0.65)$ & $0.32(0.17-0.61)$ & $0.16(0.09-0.30)$ & $0.48(0.30-0.77)$ \\
\hline & \multicolumn{2}{|l|}{-1.17} & -0.96 & -1.13 & -1.83 & \\
\hline Self-rated health & ' & ' & $41.14 x^{2}(4)^{* * *}$ & ' & ' & ' \\
\hline \multirow[t]{2}{*}{ Very good (ref) } & & & 1.00 & & & \\
\hline & & & 0 & & & \\
\hline Good & ' & ' & ' & ' & ' & ' \\
\hline \multirow[t]{3}{*}{ Fair } & ' & ' & $8.04 x^{2}(1)^{* *}$ & , & ' & ' \\
\hline & & & $0.48(0.29-0.80)$ & & & \\
\hline & & & -0.74 & & & \\
\hline \multirow[t]{3}{*}{ Bad } & ' & ' & $14.63 x^{2}(1)^{* * *}$ & ' & ' & ' \\
\hline & & & $0.27(0.14-0.53)$ & & & \\
\hline & & & -1.31 & & & \\
\hline \multirow[t]{3}{*}{ Very bad } & & & $2.44 x^{2}(1)$ & & & \\
\hline & & & $0.37(0.11-1.29)$ & & & \\
\hline & & & -1.00 & & & \\
\hline \multirow{2}{*}{$\begin{array}{l}\text { Whether respondent has } \\
\text { chronic health condition }\end{array}$} & ' & ' & ' & 1.00 & ' & ' \\
\hline & & & & 0 & & \\
\hline \multicolumn{7}{|l|}{ Yes (ref) } \\
\hline \multirow[t]{2}{*}{ No } & ' & ' & ' & $x 2(1)=6.70^{*}$ & ' & ' \\
\hline & & & & $2.51(1.25-5.04)$ & & \\
\hline
\end{tabular}

Ref: reference level, ${ }^{*} \mathrm{p}<.05 ;{ }^{* *} \mathrm{p}<.01,{ }^{* * *} \mathrm{p}<.001$.

* Levels too low in one item (widowed $f=3$; separated $f=1$ ).

** Levels too low in one item (separated $f=4$ ).

*** Levels too low in one item (self-employed $\mathrm{f}=7$ ).

\section{Sex}

In Australia, males were about $45 \%$ more likely to state that cost was not a difficulty factor on the last occasion they required to see a doctor/specialist than females [OR=0.54, CI=0.38-0.76, $\mathrm{p}<0.001]$.

Males in Korea were about $30 \%$ more likely than females $[\mathrm{OR}=0.72, \mathrm{CI}=0.54-0.97, \mathrm{p}<0.01]$ to indicate that cost was a not difficulty factor on the last occasion they required to see a doctor/specialist.

\section{Age}

In Japan, respondents who were 60 years or older were nearly twice as likely $[\mathrm{OR}=1.89, \mathrm{CI}=1.22-2.94, \mathrm{p}<0.01]$ to state that cost was not a difficulty factor on the last occasion they required to see a doctor/specialist than those who were between 20 and 29 years old.

\section{Marital status}

In Australia, respondents who had never married were about $50 \%$ more likely to state that cost was not a difficulty factor than those who were married $[\mathrm{OR}=0.48$, $\mathrm{CI}=0.30-0.77, \mathrm{p}<0.01]$.

In Hong Kong, respondents who were widowed were about 19 times more likely $[\mathrm{OR}=19.81, \mathrm{CI}=5.37-73.05$, $\mathrm{p}<0.001]$ to state that cost difficulties were not an issue on the last occasion they required to see a doctor/specialist than those who had never married.

\section{Work status}

Australians who were retired/pensioners were about 3.3 times more likely $[\mathrm{OR}=3.32, \mathrm{CI}=1.84-5.99, \mathrm{p}<0.001]$ to state cost was not a difficulty on the last occasion they required to see a doctor/specialist than full-time workers. Those who were housewives or househusbands were twice as likely [OR=2.03, $\mathrm{CI}=1.00-4.08, \mathrm{p}<0.05]$ to state cost was not a difficulty than those who were fulltime workers.

In Hong Kong, respondents who worked full-time were approximately $90 \%$ more likely to state that cost was a not difficulty factor compared with those who were retired or pensioners $[\mathrm{OR}=0.12, \mathrm{CI}=0.64-0.21$, $\mathrm{p}<0.001]$.

In Taiwan, respondents who worked full-time were about $70 \%$ more likely to state that cost was not a difficulty factor on the last occasion they required to see a doctor/specialist than those who worked part-time $[\mathrm{OR}=0.31, \mathrm{CI}=0.18-0.53, \mathrm{p}<0.01]$. 


\section{Discussion}

The aim of this manuscript is to highlight disparities and differences in terms of the factors that seem to be important to distinguishing between poor and good access to healthcare across six countries. In the following we discuss these findings in relation to healthcare provision within each of these countries. We do not provide a comprehensive explanation of our findings but rather, we provide a level of interpretation around the country context for each of the findings. Our results for each country should be used as baseline data or as the basis for an intervention by researchers with knowledge of the local, social, cultural and political context.

\section{Australia}

The findings identified that respondents who are unhappy with their health have difficulty accessing services due to distance. This is obviously worrying, and is an example of the 'inverse care law' [23]. This is of concern given the potential for increasing inequities in health outcomes.

The findings regarding females having difficulty accessing services is also concerning. Studies from both high- and low income countries show higher rates of morbidity among women than men [67]. However, in line with previous research, the findings may also provide further evidence to suggest that health services are under-utilised (thereby rendering difficulties in access irrelevant) by men which has obvious implications for men's health in Australia. Data from the UK suggest that men are less likely than women to consult their general practitioners but have higher admission rates for chronic disease which suggests that men's health policy efforts may need to focus on promotion and prevention [68].

Finances, inclusive of annual income and difficulty with finances in the past year, were also found to be a predictor of poor access to healthcare across distance, appointment, waiting time and cost. Previous research conducted in Australia identified that patients with low socioeconomic status are likely to have shorter consultations with GPs despite the fact that, as a group, they have a significantly higher need for care [69]. Although Australia has publically funded healthcare via Medicare [70], waiting times and difficulties in being granted a medical appointment continue to be a problem in Australia. Individuals have the option of purchasing private services via out of pocket payment or private insurance schemes which minimises waiting times and permits more timely access to specialists via private appointments. However, figures from the ABS 2007-08 National Health Survey indicate that only half $(53 \%)$ of the Australian population aged 15 years and over have private health insurance [71].

\section{Hong Kong}

Respondents in Hong Kong who spent their savings in the past year and who fell in the lowest income quartile were found to have difficulties accessing healthcare due to distance. We cannot explain this finding and suggest that more research is needed to understand the role of poverty in determining geographical inequity in access to services.

Older individuals (over 60, pensioners, retired) were found to have problems accessing health services do to difficulties getting appointments, waiting times and cost. At the time of data collection (the 2008-09 financial year), the Government of Hong Kong launched the Elderly Health Care Voucher Pilot Scheme (HCVS) for three years to provide five healthcare vouchers of $\$ 50$ each to elders aged 70 or above annually to partially subsidise their use of private primary healthcare (PHC) services. There was recognition by government that programs were needed for the elderly to be granted better access to care and a continuity of care from their chosen providers. The additional aim was to reduce elderly reliance on public healthcare resources so that other members of the public who are in need of primary healthcare services would be benefit indirectly. Results from an interim review on the Scheme have demonstrated its success and the Government has decided to extend the Scheme for another three years from 1 January 2012 to 31 December 2014, increasing the voucher amount entitled by each eligible elder from $\$ 250$ to $\$ 500$ per year [72]. Our recommendation is to obtain a repeat measure of access to healthcare as a means of identifying the impact of this policy on access to healthcare for the elderly.

\section{Japan}

Japan has been argued to be one of the most equitable one-tiered (solely private) health systems in the world [3,73]. Roughly $80 \%$ of Japan's hospitals and $94 \%$ of its physician-run offices (referred to as clinics) are privately operated; however all care is provided under a nationally uniform fee schedule which means that neither insurers nor providers have the freedom to negotiate individual fee schedules $[74,75]$. Every citizen must be attached to one scheme but insurance premiums are calculated on the basis of income. Individuals can be on public assistance which is the only situation under which an individual does not have to be covered by an insurance scheme.

Our results identified that difficulties in the financial year prior to data collection was predictive of difficulties in accessing healthcare due to cost. Although insurance premiums are regulated, as noted above, these premiums are calculated based on income. It is possible that healthcare premiums are calculated in a manner that does not take into account current/recent income (i.e. 
people are charged more than they can afford until income is reassessed).

Additionally, in contrast to trends across the other countries, being satisfied with one's health was a predictor of difficulties in gaining an appointment. We cannot explain this finding and suggest that more research is needed to understand if health care service access is prioritised according to need (i.e. people dissatisfied with their health are priority).

\section{Korea}

Respondents who spent savings or borrowed money in the year prior to data collection were found to have difficulty in accessing care due to cost. This may be due to the nature of the South Korean healthcare system. All Koreans are entitled to the National Health Insurance Program (NHIP); however, Song (2009) reported that only $57.7 \%$ were enrolled in this service in 2006 . Additionally, for those who do enrol, it is required that individuals pay a portion of the healthcare costs, which vary according to the level and type of healthcare institution being used. Some rates are as high as $50 \%$ of the total cost of treatment (i.e. for general hospital visits and treatments). Those who cannot afford co-payments are covered by the Medical Aid Program which pays for all medical expenses for those who are unable to pay. However, only 3.7\% of South Koreans are eligible for this service (Song 2009).

\section{Taiwan}

A National Health Insurance (NHI) scheme covers 99\% of Taiwan's population [76]. The objective of the NHI is to provide equal access to healthcare for all citizens and to maintain healthcare expenditure at manageable levels [77]. By law, regardless of age, gender, or employment status, any Taiwanese citizen with a local ID card or a foreign national living in Taiwan with an Alien Resident Certificate (ARC) is required to enrol in the program. Additionally, those eligible for the system must participate in the system (unless they lose their eligibility by being convicted of a crime, disappearing, giving up their Taiwan citizenship, moving abroad or having their Alien Resident Certificate expired) [78]. The premium for the insurance scheme is $100 \%$ subsidized for households below the poverty line. Despite this, we identified that difficulties in finances in the year prior to data collection and working part time were predictors of difficulties in access due to cost. Our findings may be explained by the fact that part-time workers or individuals who had difficulty in the financial year prior to data collection may not be eligible for subsidized premiums [79]. While the scheme is designed so that no single individual living in Taiwan will be denied healthcare for lack of means, low- to-middle income earners may fall short of eligibility criteria for access to these subsides.

\section{Thailand}

Employment status was the main predictor for difficulty in accessing healthcare in Thailand. Individuals who work for their family, do part time work, work within the household or are unemployed were found to have difficulty accessing services due to distance, difficulties getting an appointment and/or waiting times. This may be explained in part by the structure of the Thai social health insurance program. Thai individuals working in the private sector are provided with health insurance under the Civil Servant Medical Benefit Scheme (CSMBS) or the Social Security Scheme. However, this social insurance scheme does not cover people working outside of the private sector, non-working spouses, child dependents or other family members. While there is a scheme used by the remainder of the population, referred to as the 30-Baht Scheme whereby a person pays 30 Baht to receive access to any health service required, Thailand still struggles to extend coverage to the majority of its population [80]. The 30-Baht Scheme may also explain the finding that respondents who had financial difficulties in the year prior to the data collection also found it difficult to access service due to cost, whereby some individuals may not be able to afford this prescribed payment.

Consistent across the findings, poor health and low income were identified as difficulties in access due to distance. In a similar vein, low income was found to be a predictor for difficulties in access due to cost. These findings echo previous cross-country research investigating access (Burkina Faso, Guatemala, Kazakhstan, Kyrgyzstan, Paraguay, South Africa, Thailand, Zambia), whereby it was found that wealthier population groups have a higher probability of obtaining healthcare when they need it, are more likely to be seen by a doctor, and to receive medicines [81]. Our findings present further evidence of the inverse care law - population groups with the highest levels of healthcare need (economically deprived, poor health status) often have the poorest access to services [23]. The findings suggest that regionally (Asia-Pacific), policy makers need to recognise the increasing inequities for these population groups and react accordingly which is inclusive of addressing the foundations of these inequities through policy/system change.

Excluding poor health and income, the picture is more complex when looking at trends in predictors within countries. For Thailand, the poorest level of access appears to be for respondents who work within the household whereas in Taiwan, part-time work is associated with difficulties in access. Within Hong Kong, results suggest that older (above 60) and retired individuals 
have the poorest access and within Australia, females and married individuals are the worst off. The countries involved in this study are in varying stages of socioeconomic and health development. The needs and functions of each country's healthcare system therefore vary and require policy reform tailored to the contours of their health systems. However, some conclusions can be drawn from the successes of these countries. While we identified health access to be problematic for older individuals in Hong Kong, recent policy has shifted to increase access to services for older individuals which indirectly, has been suggested to be delivering more equitable service for the remainder of citizens. Likewise, Taiwan has developed a system which was specifically designed to provide universal services for all individuals. Our findings also identify the need for national policies to address country-specific inequities.

\section{Conclusion}

It is recognised trans-nationally that public health policy and practice needs to focus on addressing the Social Determinants of Health in order to increase the health of the most vulnerable and disadvantaged groups [5]. This research has identified a number of social determinants which predict inequities in access to healthcare in six countries across Asia-Pacific. We have discussed our findings in relation to the political climate of healthcare in each of the six countries.

Recognition of these inequities, from a policy perspective, is essential for health sector policy decision-making [81]. Health policies shape health systems, and consequently, the broader determinants of health [82]. These policies should be informed by an understanding of the difficulties experienced by individuals when trying to obtain medical attention [36]. Despite the differences in political and economic climate in the countries under analysis, our findings highlight patterns of inequity which require policy responses. We argue that our data should be used as a means of deciding the most appropriate policy response for each country which includes, rather than excludes, socially marginalised population groups [83]. These findings should be of interest to those involved in health policy, but also in policy more generally because as we have identified, health is influenced by determinants outside of the health system [84].

Our results also provide baseline measures for identifying where and how policy should be altered to enable equitable access. Furthermore, as suggested for reforms in Hong Kong, these measures should be used for future policy evaluation to identify if shifts in policy have indeed improved access for the marginalised groups identified. An investigation into the possible causes of these inequities, and plans to address them, should be the focus of government agendas.
At this point, the authors wish to recognise and briefly discuss potential shortcomings stemming from aspects of the design, data collection and analytical procedures associated with this research. As presented in the results section, the amount of variance explained by the factors included in the models specified for all countries separately and combined was generally small as indicated by low Nagelkerke R2 estimates. It is therefore acknowledged that the (socio) demographic predictors included in the current investigation do not achieve substantial contributions to explaining response differences, hence further variables need to be investigated which might carry stronger predictive qualities for the outcome variables examined. Despite the comparatively small amount of variance explained, the models specified were (highly) significant which is taken as being indicative of small, yet significant contributions of (socio) demographic variables to various aspects of access to healthcare.

It is furthermore acknowledged that differences in survey format, the exact formulation of individual survey items and response options, as well as data collection in the different settings have potentially introduced biases when aggregating data and/or comparing the results. Maintaining data integrity and keeping the data collected as close to their raw form as possible meant particular predictor as well as outcome variable levels had to be collapsed and comparisons drawn between variables which are not $100 \%$ identical in terms of meaning, response format, etc. Several problems arise from this process, including the loss of fine-grained differences for certain predictor and outcome variable levels which may have resulted in slightly different make-up of significant regression models. Whilst this undoubtedly creates limitations for the extent to which the current findings can be generalised, this study presents the first attempts to conduct a secondary analysis of cross-country data collected for this specific purpose using study materials which have been generated with the aim to be applicable in different settings. The authors therefore acknowledge that limitations apply which mean the current results need to be interpreted and generalised with caution, and that replication is required to strengthen the existing body of evidence. Nevertheless, given the relative paucity of data in this area of interest, the current results add substantially to our current understanding of variables affecting access to healthcare in different countries.

\section{Endnotes}

${ }^{1}$ The broader study from which the data in this manuscript emanate was based on Social Quality Theory (SQT). We have provided detailed manuscripts on SQT elsewhere Within Social Quality Theory, access to healthcare is identified as an indicator of social quality [52]. 
${ }^{2}$ Japan (Chiba University), Taiwan (National Taiwan University), Hong Kong (Chinese University Hong Kong), Thailand (King Prajadhipok Institute), Korea (Seoul National University).

${ }^{3}$ The Japanese sample was not stratified according to health insurance type which may have implications for the interpretation of the Japanese data.

\section{Competing interests}

The authors declare that they have no competing interests.

\section{Authors' contributions}

CNL and SBM participated in the design of the study, piloting and data collection. CNL and LM carried out the data analysis. All authors drafted the manuscript. PRW wrote the grant application and received funding for the study. All authors read and approved the final manuscript.

\section{Acknowledgements \\ We would like to thank members of the Asian Social Quality Network for assisting in developing the survey on which this manuscript is based. In particular, we would like to thank Prof Jaeyeol Yee and Prof Dukjin Chang from Seoul National University, Korea for driving the development of the survey. We would also like to thank those involved in the data collection in each of the participating countries and specifically, Prof Tetsuo Ogawa (Japan, Chiba University), Prof Lillian Wang (Taiwan, National Taiwan University), Prof Raymond Chan (Hong Kong, Chinese University Hong Kong), Prof Thawilwadee Bureekul (Thailand, King Prajadhipok Institute), Prof Dukjin Chang (South Korea, Seoul National University). Additionally we would like to acknowledge Flinders University Faculty of Health Sciences for funding this research via a faculty seeding grant.}

Received: 23 October 2012 Accepted: 21 June 2013

Published: 1 July 2013

\section{References}

1. Commission on Social Determinants of Health: Closing the gap in a generation: Health equity through action on the social determinants of health. In Final report to the CSDH. Geneva: World Health Organisation; 2008.

2. Pittnam PM: Beyond the sound of one hand clapping: experiences in six countries using health equity research in policy. I Health Polit Policy Law 2006, 31:33-49.

3. Gilson L, Doherty J, Loewenson R, Francis V: Final Report: Knowledge Network on Health Systems, Challenging Inequity Through Health Systems. WHO Commission on Social Determinants of Health; 2007. http://www.who.int/ social_determinants/resources/csdh_media/hskn_final_2007_en.pdf

4. National Preventative Health Taskforce: Australia: the healthiest country by 2020. A discussion paper. Canberra: Commonwealth of Australia; 2008.

5. Ward PR, Meyer SM, Verity F, Gill TK, Luong TCN: Complex problems require complex solutions: the utility of social quality theory for addressing the social determinants of health. BMC Publ Health 2011, 11:630.

6. WHO: Jakarta Declaration on Leading Health Promotion into the 21st Century. Geneva: WHO; 1997

7. WHO: Geneva. Geneva: WHO; 2005.

8. Kondro W: The fiendish puzzle of health inequities. Can Med Assoc J 2012, 184(13):1456-1457.

9. Ostlin P, Schrecker T, Sadana R, Bonnefoy J, Gilson L, Hertzman C, Kelly M, Kjellstrom T, Labonte R, Lundberg $\mathrm{O}$, et al: Priorities for research on equity and health: implications for global and national priority setting and the role of WHO to take the health equity research agenda forward. Geneva: World Health Organization; 2010.

10. Commission on Social Determinants of Health: Action on the Social Determinants of Health: Learning from previous experiences. Geneva: World Health Organisation; 2005.

11. Commission on Social Determinants of Health: Achieving health equity: from root causes to fair outcomes. Geneva: World Health Organisation; 2007.
12. Commission on Social Determinants of Health: Closing the gap in a generation: Health equity through action on the social determinants of health. Final report of the CSDH. Geneva: World Health Organisation; 2008.

13. Javanparast S, Ward PR, Carter S, Wilson C: Barriers to and facilitators of colorectal cancer screening in different population sub-groups in Adelaide South Australia. Med J Aust 2012, 196:521-523.

14. McCaffery K, Wardle J, Nadel M, Atkin W: Socioeconomic variation in participation in colorectal cancer screening. J Med Screen 2002, 9:104-108.

15. Ward PR, Javanparast S, Cole S, Gill T, Ah Matt M, Aylward P, Baratiny G, Jiwa M, Martini A, Misan G, et al: The equity of colorectal cancer screening: a cross-sectional analysis of the National Bowel Cancer Screening Program data for South Australia. Aust N Z J Public Health 2011, 35:61-65.

16. Ward PR, Javanparast $S$, Wilson C: Equity of colorectal cancer screening: which groups have inequitable participation and what can we do about it? Aust J Prim Health 2011, 17:334-346.

17. Battersby J, Flowers J, Harvey I: An alternative approach to quantifying and addressing inequity in healthcare provision: access to surgery for lung cancer in the east of England. J Epidemiol Community Health 2004 58:623-625.

18. Hippisley-Cox J, Pringle M: Inequities in access to coronary angiography and revascularisation: the association of deprivation and location of primary care services. Br J Gen Pract 2000, 50(455):449-454.

19. Majeed A, Eliahoo J, Bardsley M, Morgan D, Bindman AB: Variation in coronary artery bypass grafting, angioplasty, cataract surgery, and hip replacement rates among primary care groups in London: association with population and practice characteristics. J Public Health Med 2002, 24:21-26.

20. Hull SA, Cornwell J, Harvey C, Eldridge S, Bare PO: Prescribing rates for psychotropic medication amongst east London general practices: low rates where Asian populations are greatest. Fam Pract 2001, 18:167-173.

21. Ward PR, Noyce PR, St Leger AS: Exploring the equity of GP practice prescribing rates for selected coronary heart disease drugs: a multiple regression analysis with proxies of health care need. International Journal for Equity in Health 2005, 4(3). doi:10.1186/1475-9276-4-3

22. Ward PR, Noyce PR, St Leger AS: How equitable are GP practice prescribing rates for statins?: an ecological study in four primary care trusts in the North West of England. International Journal for Equity in Health 2007, 6(2). doi:10.1186/1475-9276-6-2

23. Tudor Hart J: The inverse care law. Lancet 1971, 297(7696):405-412.

24. Dahlgren G, Whitehead M: Levelling up (Part 2): a discussion on European strategies for tackling social inequities in health. Copenhagen: WHO Regional Office for Europe; 2006

25. Whitehead M, Dahlgren G: Levelling up (Part 1): a discussion paper on concepts and principles for tackling social inequities in health. Copenhagen: WHO Regional Office for Europe; 2006.

26. McMurray A: Community health and wellness: A sociological approach. Sydney: Mosby Publishers; 1999.

27. Braveman P: Health disparities and health equity: concepts and measurement. Annu Rev Public Health 2006, 27:167-194.

28. Macinko JA, Starfield B: Annotated bibliography on equity in health, 1980-2001. International Journal for Equity in Health 2002, 1. doi:10.1186/ 1475-9276-1-1

29. Sen A: Why Health Equity? In Public Health, Ethics, and Equity. Edited by Anand S, Fabienne P, Sen A. Oxford: Oxford University Press; 2006.

30. Starfield B: State of the art in research on equity in health. $J$ Health Polit Policy Law 2006, 31:11-32.

31. Goddard M, Smith P: Equity of access to health care services: theory and evidence from the UK. Soc Sci Med 2001, 53:1149-1162.

32. Oliver A, Missialos E: Equity of access to health care: outlining the foundations for action. J Epidemiol Community Health 2004, 58:655-658.

33. Ward PR: Equity in access to healthcare services - a social determinants of health. In Understanding Health - A Determinants Approach. Edited by Keleher H, MacDougall C. Oxford: Oxford University Press; 2009.

34. Ward PR, Noyce PR, St Leger AS: Are GP practice prescribing rates for CHD drugs equitable? A cross-sectional analysis in four primary care trusts in England. J Epidemiol Community Health 2004, 58:89-96.

35. Braveman P: Monitoring equity in health and healthcare: a conceptual framework. J Heal Popul Nutr 2003, 21(3):181-192.

36. Gulliford M, Figueroa-Munoz J, Morgan M, Hughes D, Gibson B, Beech R Hudson M: What does 'access to health care' mean? Journal of Health Services Research \& Policy 2002, 7(3):186-188. 
37. Braveman $P$, Gruskin S: Defining equity in health. J Epidemiol Community Health 2003, 57:254-258.

38. Aday LA, Anderson RM: Equity of access to medical care: a conceptual and empirical overview. Medical Care 1981, 19(suppl):4-27.

39. Culyer AJ, Wagstaff A: Equity and equality in health and health care. $J$ Heal Econ 1993, 12:431-457.

40. Mooney GH: Is it not time for health economists to rethink equity and access? J Health Polit Policy Law 2009, 4:209-221.

41. Mooney GH, Hall J, Donaldson C, Gerard K: Utilisation as a measure of equity: weighing heat? J Heal Econ 1991, 10(4):475-480.

42. Mooney GH: Equity in health care: confronting the confusion. Effective Health Care 1983, 1:179-185.

43. Culyer AJ, Doorslaer EV, Wagstaff A: Comment: utilization as a measure of equity by Mooney, Hall, Donaldson and Gerard. J Heal Econ 1992, 11(1):93-98.

44. Dixon-Woods M, Cavers D, Agarwal S, Annandale E, Arthur A, Harvey J, Hsu R, Katbamna S, Olsen R, Smith L, et al: Conducting a critical interpretive synthesis of the literature on access to healthcare by vulnerable groups. BMC Med Res Methodol 2006, 6(35). doi:10.1186/1471-2288-6-35

45. Glossary of frequently encountered terms in health economics. http://www. nlm.nih.gov/nichsr/edu/healthecon/glossary.html

46. Accessing health care: responding to diversity. Edited by Healy J, McKee M. Oxford: Oxford University Press; 2004.

47. Bower P, Roland M, Campbell J, Mead N: Setting standards based on patients' views on access and continuity: secondary analysis of data from the general practice assessment survey. Br Med J 2003, 326(7383):258-262

48. Turnock BJ: What it is and How It Works. London, UK: Jones and Bartlett Publishers; 2009

49. Aday $L A$, Andersen $R$ : $A$ framework for the study of access to medical care. Heal Serv Res 1974, 9(3):208-220.

50. Allin S, Masseria C, Mossialos E: Measuring socioeconomic differences in use of health care services by wealth versus by income. Am J Public Health 2009, 99(10):1849-1855.

51. Dixon A, Le-Grane J, Henderson J, Murray R, Poteliakhoff E: Is the NHS equitable? A review of the evidence. London: LSE Health and Social Care, The London School of Economiocs and Political Science; 2003.

52. van der-Maesen $L J G$, Walker A: Indicators of Social Quality: outcomes of the European scientific network. Eur J Soc Qual 2005, 5(1/2):8-24

53. Meyer SB, Luong CN, Tsourtos G, Ward PR: Operationalising the theory of social quality: analysis of the reliability of an instrument to measure social quality. Development and Society 2010, 39(2):327-356

54. Chen F-I, Shi S-J: Social exclusion experiences of atypical workers: a case study of Taipei. International Journal of Social Quality 2013, 2(2). http://dx. doi.org/10.3167/IJSQ.2012.020204

55. Vajirakachorn S: Social inclusion in Southern Border Provinces of Thailand. International Journal of Social Quality 2013, 2(2). http://dx.doi.org/ 10.3167/IJSQ.2012.020205

56. Hu K, Chan RKH: Social capital and civic engagement in urban China. International Journal of Social Quality 2013, 2(2). http://dx.doi.org/10.3167/ IJSQ.2012.020203.

57. Bureekul T, Thananithichot S: Trust and Social Cohesion, the Key to Reconcile Thailand Future. Quality: International Journal of Social; 2013:2(2).

58. Adamson J, Ben-Schlomo Y, Chaturvendi N, Donovan J: Ethnicity, socio-economic position and gender - do they affect reported health-care seeking behaviour? Soc Sci Med 2003, 57:895-904.

59. Lu JR, Leung GM, Kwon S, Tin KYK, Doorslaer EV, O'Donnell O: Horizontal equity in health care utilization evidence from three high-income Asian economies. Soc Sci Med 2007, 64:166-212

60. Hosmer DW, Lemeshow S: Applied Logistic Regression. 2nd edition. New Jersey: John Wiley \& Sons; 2000

61. Nagelkerke NJD: A note on a general definition of the coefficient of determination. Biometrika 1991, 78(3):691-692

62. Field A: Discovering Statistics Using SPSS. 3rd edition. London: Sage; 2009.

63. O'Brien RM: A causation regarding rules of thumb for variance inflation factors. Qual Quant 2007, 41(5):673-690.

64. Rice N, Robone S, Smith PC: International comparison of public sector performance: the use of anchoring vignettes to adjust self-reported data. Evaluation 2010, 16(1):81-101.

65. Salomon J, Tandon A, Murray CJ: Comparability of self-rated health: corss sectional multi-country survey using anchoring vignettes. Br Med J 2004 328(7434):258
66. Vermunt JK: Mixed-effects logistic regression models for indirectly observed discrete outcome variables. Multivariate Behavioural Research 2005, 40(3):281-301

67. Sen G, Ostlin P, George A: Unequal, unfair, ineffective and inefficient gender inequity in health: why it exists and how we can change it. In Ineffective and Inefficient Gender Inequity in Health: Why it exists and how we can change it. Women and Gender Equity Knowledge Network, WHO Commission on Social Determinants of Health; 2007. http://www.who.int/ social_determinants/resources/csdh_media/wgekn_final_report_07.pdf

68. Griffiths S: Men's health: unhealthy lifestyle and an unwillingness to seek medical help. BMJ 1997, 312(13):69-70.

69. Furler JS, Harris E, Chondros P, Powell Davies PG, Harris MF, Young DYL: The inverse care law revisited: impact of disadvantaged location on accessing longer GP consultation times. Med J Aust 2002, 177:80-83.

70. Willis EM, Reynolds L, Keleher H: Understanding the Australian Health Care system. Chatswood NSW: Churchill Livingstone Elsevier; 2009.

71. Health Services: Use and Patient Experience. [http://www.abs.gov.au/ AUSSTATS/abs@.nsf/Lookup/4102.0Main+Features20Mar+2011]

72. Background of Elderly Health Care Voucher Pilot Scheme [http://www.hcv.gov. hk/text/eng/pub_background.htm]

73. Ikegami N: Japanese health care: low cost through regulated fees. Heal Aff 1991, 10(3):87-109.

74. Reid TR: The Healing of America: A Global Quest for Better, Cheaper, and Fairer Health Care. New York: Penguin Books; 2010

75. Campbell JC, Ikegami N: The Art of Balance in Health Policy: Maintaining Japan's Low-cost Egalitarian System. Cambridge, UK: Cambridge University Press; 1998.

76. International Symposium on Achievements and Challenges of National Health Systems: To celebrate the 10th anniversary of Taiwan's National Health Insurance. Taipei, Taiwan; 2005.

77. Leung GM, Wagstaff A, Lindelow M, Lu JR: Health Systems of China, Hong Kong and Taiwan. In International Encyclopaedia of Public Health Volume 4. Edited by Heggenhougen HK, Quah S. San Diego CA: Academic Press; 2008:656-671.

78. Program overview [http://www.nhi.gov.tw/English/webdata/webdata.aspx? menu=11\&menu_id=590\&WD_ID=590\&webdata_id=3139]

79. Universal Health Coverage in Taiwan [http://www.nhi.gov.tw/Resource/ webdata/21717 1_20120808UniversalHealthCoverage.pdf

80. Caussy D, Sein T: Health systems of East Asia and Pacific States. In International Encyclopaedia of Public Health Volume 4. Edited by Heggenhougen HK, Quah S. San Diego CA: Academic Press; 2008:267-276.

81. Makinen M, Waters H, Rauch M, Almagambetova N, Bitran R, Gilson L, Mclntyre D, Pannarunothai S, Prieto AL, Ubilla G, et al: Inequalities in health care use and expenditures: empirical data from eight developing countries and countries in transition. Bull World Health Organ 2000, 78(1):55-65.

82. What is HSPR? Overview. http://www.who.int/alliance-hpsr/about/hpsr/en/ index.html

83. Freedman LP, Waldman RJ, de-Pinho H, Wirth ME, Chowdhury AMR, Rosenfield A: UN Millennium Project Task Force on Child Health and Maternal Health, Who's got the power? Transforming health systems for women and children. London: Earthscan; 2005.

84. Lee K, Fustukian S, Buse K: An introduction to global health policy. In Health Policy in a Globalising World. Edited by Lee K, Buse K, Fustukian S. Cambridge: Cambridge University Press; 2002.

doi:10.1186/1472-6963-13-238

Cite this article as: Meyer et al:: Inequities in access to healthcare: analysis of national survey data across six Asia-Pacific countries. BMC Health Services Research 2013 13:238. 